
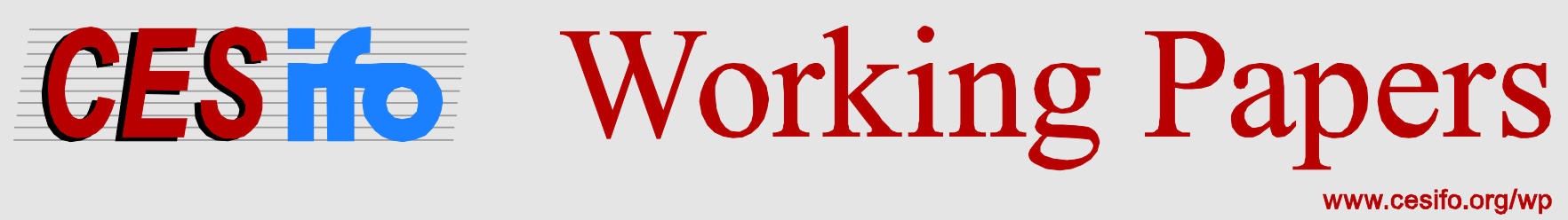

\title{
Constraints on Matching Markets Based on Moral Concerns
}

\author{
Katharina Huesmann \\ Achim Wambach
}
CESIFO WORKING PAPER NO. 5356
CATEgORY 2: PUBLIC CHOICE
MAY 2015
An electronic version of the paper may be downloaded
- from the SSRN website:
- from the RePEc website:
- from the CESifo website:
www.SSRN.com
Www.RePEc.org
www.CESifo-group.org/wp




\title{
Constraints on Matching Markets Based on Moral Concerns
}

\begin{abstract}
Various markets ban or heavily restrict monetary transfers. This is often motivated by moral concerns. However, it appears to be disputable whether the observed restrictions on transfers are the appropriate market design answer to these concerns. Instead of exogenously restricting transfers on a matching market, we introduce a desideratum based on fairness objectives and study its market design implications. The desideratum we concentrate on is discriminationfreeness, i.e. one's access to certain resources is independent of one's wealth endowment. A key assumption in our model is that preferences are not quasilinear but wealth has an impact on the willingness to pay. We show that matchings without transfers based on ordinal object rankings are at the efficient frontier of discrimination-free social choice functions. Implementable social choice functions are discrimination-free if and only if an agent's object assignment only depends on ordinal object rankings and her money assignment is constant. If money can be used outside the market designer's control even externality-freeness is needed: an agent's object assignment has to be independent of other agents' types. We discuss several applications in the context of discrimination-freeness including compensation for kidney donors.
\end{abstract}

JEL-Code: D470, D630, I000, D610, D820.

Keywords: repugnance, inequality, market design, matching markets.

Katharina Huesmann

Cologne Graduate School in Management, Economics and Social Sciences

Cologne / Germany

huesmann@wiso.uni-koeln.de
Achim Wambach

University of Cologne

Department of Economics

Cologne / Germany

wambach@wiso.uni-koeln.de

May 11, 2015

We especially thank Felix Bierbrauer for insightful discussions. We furthermore thank Sophie Bade, Philipp Heller, Hartmut Kliemt, Ulrike Malmendier, Timo Mennle, Al Roth, and seminar and conference participants in Berlin, Cologne, at SMYE 2012, "Frontiers of Market Design" 2012, EEA-ESEM 2012, Summer School on Matching Problems, Markets and Mechanisms 2013, and $25^{\text {th }}$ Summer School in Economic Theory. 


\section{Introduction}

Why worry that we are moving toward a society in which everything is up for sale? For two reasons. One is about inequality, the other about corruption. [...] Where all good things are bought and sold, having money makes all the difference in the world.

Michael Sandel in "What Money Can't Buy", 2012

Various markets ban monetary transfers or heavily regulate them. Especially education and health markets provide many examples. School or university places must not be traded for money in many countries. Several countries do not charge fees for school or university places but fund them via taxes. Almost everywhere in the world selling organs or financially compensating organ donations is prohibited.

If maximizing efficiency is the goal, it appears irrational from an economic perspective to prohibit transactions that have no negative externalities. Indeed, views on whether to allow transfers are not unanimous. In the US, compensations for kidney donors are currently intensively discussed. Proposals go from free markets over regulated markets to strictly prohibiting any monetary transfers, as it is current practice. ${ }^{1}$ Other examples of intense public and political discussions include tuition fees. In Germany, after a period of having (basically) not charged any fees, from 2006 on universities were allowed to charge up to 500 EUR per semester. Protests were huge and finally, in 2014, there is no public university left charging fees. ${ }^{2}$

The economic market design literature dealing with the question whether to admit money or not is scarce. A large branch of research discusses how to allocate resources if the absence of transfers is an exogenous constraint. ${ }^{3}$ Furthermore, several works exist on how introducing money might effect resource supply. ${ }^{4}$ However, ethics behind the absence of transfers are barely discussed and money appears to be considered as a taboo in certain markets. Roth (2007) introduces the concept of repugnance and illustrates that in some markets repugnance has established real constraints, such as the absence of monetary transfers.

On the other hand, numerous philosophical essays discuss the ethics of money. A main concern that appears to drive the discussion about noxious markets is inequality. It is feared that differences in wealth determine the access to certain goods once

\footnotetext{
${ }^{1}$ See, e.g., http://marketdesigner.blogspot.co.il/ for an overview of the discussion.

${ }^{2}$ http://theconversation.com/how-germany-managed-to-abolish-university-tuition-fees-32529

${ }^{3}$ For a comprehensive matching markets survey see, for instance, Sönmez and Unver (2011).

${ }^{4}$ Becker and Elias (2007), for instance, argue that monetary incentives could solve the shortage of available kidney donors. Richard (1970) and subsequent literature, e.g. Frey and OberholzerGee (1997), Mellström and Johannesson (2008), and Gneezy and Rustichini (2000), discuss to what extend money might lead to decreasing supply due to crowding out altruism.
} 
a market is in place, then resulting in unfair living conditions and opportunities. Furthermore, financially distressed persons might be open to exploitation and coercion once money is involved since they are not completely free in their choice. Satz (2010) and Sandel (2012), for instance, provide comprehensive discussions about inequality in the context of moral limits of markets.

Consensus about moral concerns appears to be much broader than consensus about market design implications. Scholars from several fields as well as practitioners discuss if banning transfers is the appropriate answer to the concerns. For instance, an open letter of October 2014 to Barack Obama and others signed by various scholars criticizes and challenges the wide-spread assumption that on many markets the existence of transfers is equivalent to a violation of associated moral concerns. ${ }^{5}$

Evidently, there is a need to understand more deeply how to address moral concerns associated with transfers in market design applications. Our paper takes a first step in this direction. Instead of exogenously banning transfers when assigning discrete resources we require a new notion of fairness that is based on a desire for a distribution of goods irrespective of financial status. Particularly, we require that somebody's resource assignment is independent of her wealth endowment. This fairness desideratum we call discrimination-freeness. Thus, if two people differ in their willingness to pay just because they differ in their wealth, discrimination-freeness requires disregarding such differences. On the other hand, if both are equally wealthy but differ in their appreciation for the objects, the differences might be exploited. ${ }^{6}$ Note that, even for equal wealth conditions, markets still might entail moral issues. However, these cases are not in our focus here, we rather consider our approach as a first step to face the issue of noxious markets.

In this paper, we analyze the market design implications of taking discriminationfreeness as a constraint in a setting where discrete resources are to be distributed and preferences are not quasi-linear in wealth. Particularly, we ask: Can transfers be used to Pareto-improve assignments without money while ensuring discriminationfreeness? What are necessary and sufficient conditions on assignments to meet discrimination-freenesss? To our knowledge, this is the first paper in this spirit. We show that transfers cannot be used to Pareto-improve a money-free assignment of discrete resources without causing discrimination. If preferences and wealth endowments are unknown, in order to ensure implementability and discrimination-freeness,

\footnotetext{
${ }^{5}$ See http://www.ustransplantopenletter.org/openletter.html.

${ }^{6}$ Our definition of discrimination-freeness appears to be in line with what people judge to be immoral based on Ambuhl et al. (2015). They present a basic model based on their survey results assuming that people judge a transaction as immoral if, from their financial perspective, they would not take part in the transaction. In their context, our definition of discrimination-freeness then generally speaking translates to requiring moral approval from anyone's financial perspective.
} 
it is a necessary condition that transfers are preference-independent and that resource assignments only depend on rank order lists. Hence, inefficiencies in markets without transfers are obtained as second best outcomes. Information on preferences beyond ordinalities can only be exploited if wealth endowments are known and ex-post wealth is made constant. For markets with discrimination-freeness as a concern, our results lay a foundation for either decoupling resource assignments and payments (as it can be observed in matching markets applications) or adjusting wealth in such a way that differences in wealth endowments are eliminated. However, we also show that banning transfers might not be sufficient to ensure discrimination-freeness. This is relevant, if money can be used outside a centralized system, for instance when a private market co-exists or when some kind of bribing occurs.

Notably, the desire to eliminate discrimination does not appear to be equally strong in all markets. Scientific and public discussions show that it seems to be especially important in markets where the good is considered necessary for developing human capital such as education and health services. In most markets for consumer goods such as cars, houses etc. the fact that the wealthy have better access is barely questioned. In many countries, access to education and health services even is a fundamental right. Leaving the individuals' well-being perspective aside and considering a country's economy, there might be further reasons why to desire no discrimination. Todaro and Smith (2003), for instance, describe the critical role of education and health for growth and development. In the following, our work does not further elaborate on the questions on what markets discrimination-freeness is especially desirable and why this is so, but rather analyzes implications for the market design if discrimination-freeness is a desideratum.

Model. We consider an allocation problem where a set of indivisible objects is to be assigned to a set of agents and each agent receives at most one object. Each agent is endowed with a type describing her initial wealth endowment $e_{i}$ and preferences $\theta_{i}$ over bundles $\left(\omega, A_{i}\right)$, where $\omega$ is an object and $A_{i}$ the total wealth of agent $i$. We consider social choice functions assigning an object and, in contrast to classical matching market models, monetary transfers to each type profile of agents. Instead of exogenously banning transfers, we introduce discrimination-freeness as a desideratum: A social choice function is discrimination-free, if the object assignment of an agent does not depend on her wealth endowment. In our analysis we are primarily interested in efficiency consequences of discrimination-freeness as well as in necessary and sufficient conditions for a social choice function to be discrimination-free and implementable in incomplete information settings.

A key assumption is that preferences are not quasi-linear. This is crucial for our 
analysis and is in contrast to many mechanism design models. Only because wealth seems to have an impact on the willingness to pay, concerns about discrimination appear. We assume that each agent's ranking of objects is wealth-independent ${ }^{7}$. However, for any two objects the willingness to pay to receive the preferred one instead of the less preferred one becomes arbitrarily low or high if the agent looses or gains wealth. ${ }^{8}$ We thus capture in our model the idea that gaining wealth results in a higher willingness to pay for a more desired object (and vice versa), while the notion of what is a more desired object does not change with wealth. A high willingness to pay for an object, therefore, can be both due to a high utility associated with the object and to high wealth. Discrimination-freeness then implies that wealth effects must not matter.

Results. First, we show that social choice functions which do not adjust wealth (i.e. functions which neither bound nor redistribute an agent's wealth endowment) cannot be Pareto-improved by using transfers without violating discrimination-freeness. Particularly, ordinal object assignments without transfers are already at the efficient frontier of discrimination-free social choice functions. Drivers of the result are that, on the one hand, the amount of money that compensates an agent for a worse object has to become larger if the agent gains wealth and, on the other hand, that the money all other agents are willing to pay for an object improvement is bounded. We show that efficient (i.e. there is no Pareto-improvement without extending the budget) and discrimination-free social choice functions exist. However, any social choice function that is discrimination-free and efficient has to adjust wealth, for instance by adjusting the wealth of each agent to a constant level.

Secondly, we show that in a setting where types are private information, an implementable social choice function is discrimination-free if and only if an agent's transfer is type-independent and an agent's object assignment only depends on her ordinal ranking of objects. Again, the income effects are crucial: If types influence transfers, there exist such preferences that in case of high wealth levels an agent with those preferences focuses on the object assignment while in case of low wealth levels the agent focuses on the monetary difference. This induces an incentive to misreport her type for low wealth levels. Serial Dictatorship, where one agent after another selects an object, is a mechanism that is implementable and at the efficient frontier

\footnotetext{
${ }^{7}$ Thus, for which objects agents compete, is independent of their wealth. Otherwise, the moral issues occurring would rather belong to segregation concerns that are not further considered here. We briefly discuss dropping the assumption of non-constant rankings as an extension.

${ }^{8}$ We show as an extension that the results only have to be slightly adjusted when modifying this assumption for instance by considering a minimum willingness to pay for object improvements.
} 
of discrimination-free social choice functions. ${ }^{9}$

Thirdly, we show that, if wealth endowments are public information, a social choice function can only exploit information about preferences that go beyond ordinal rankings if wealth is adjusted in such a way that ex-post wealth is independent of the initial wealth endowment. Otherwise, analogously to the case where the whole type is unknown, implementability and discrimination-freeness implies that object assignments are ordinal and transfers are preference-independent (but might depend on wealth). Examples for preference-independent but wealth-dependent transfers are goods that are financed via taxes and the consumption of which does not require any additional fee.

Finally, we discuss implications in the context of discrimination-freeness, if money can be used outside a mechanism designer's control to corrupt parameters of a mechanism. Using a money-free mechanism then might not be sufficient to satisfy discrimination-freeness. Technically, we extend our model to the possibility that somebody bribes somebody else to misreport preferences. Externality-freeness (i.e. an agent's assignment must not depend on other agents' preferences) is then sufficient to ensure preserving discrimination-freeness under bribes. For non-bossy social choice functions (i.e. if one cannot change another person's assignment without changing the own), externality-freeness is also necessary to ensure that discrimination-freeness under bribes is preserved. This is because bribing opportunities appear as soon as other agents' preferences play a role for an agent's assignment, but they disappear with (sufficiently large) wealth changes of one of the agents. This significantly impacts efficiency limits. If there are exactly as many objects as agents only fixed allocations such as lotteries are admissible, i.e. the assignment does not depend any more on anyone's preferences. If more objects than agents exist, the matching is wasteful, i.e. there is such a type profile that an agent prefers an unassigned object over her assignment. Bribes can be interpreted as a generalization of using money outside a centralized mechanism to influence one's access to a good. Applications include coexisting private markets or priority parameters like living in a school's neighborhood where money can help to improve one's priority.

Implications. Our results have several relevant applications. They justify the decoupling of the object assignment and the funding of resources if discrimination is a concern. This is because, on the one hand, ordinal object assignments can al-

\footnotetext{
${ }^{9}$ Serial Dictatorship is not efficient (within the set of all social choice function when transfers are allowed). However, this does not contradict the classical Gibbard-Satterthwaite Theorem (Gibbard (1973), Satterthwaite (1975)) which implies that a strategy-proof social choice function that reaches all outcomes is efficient. We restrict out attention to discrimination-free social choice function and therefore not all outcomes can be reached.
} 
ready reach the efficient frontier of discrimination-free mechanisms. On the other hand, strategy-proof object assignments with no or constant transfers are the only discrimination-free mechanisms that are implementable. Assigning the objects then is a classical matching market problem, while funding might be solved via taxes or lump-sum payments. However, our results also imply that if wealth information is available and agents' wealth is made constant, mechanisms might exploit information about cardinalities, e.g. expressed by the willingness to pay, without violating discrimination-freeness. The extension to outside options entails as a special case a classical seller and buyer model. This is relevant in several applications such as the question of whether or not to admit kidney sales. To avoid discrimination, prices have to be such that the decision, whether the transaction occurs or not, does not depend on the seller's or buyer's wealth.

Outlook. The rest of the paper is organized as follows. The next section describes the basic model. In section 3 we introduce discrimination-free social choice functions. We discuss implications of discrimination-freeness on efficiency and characterize discrimination-free social choice functions. We discuss several extensions of our basic model: two-sided markets, modifications of the type space and outside options. Section 4 considers consequences for discrimination-free social choice functions if money is used out of a mechanism designer's control. Section 5 concludes.

\section{Model}

We consider a set $N$ of $n \geq 2$ agents and a set $\Omega$ of $k \geq n$ distinct objects. Each agent is endowed with a type $t_{i}=\left(\theta_{i}, e_{i}\right) . e_{i} \in \mathbb{R}$ describes agent $i$ 's initial wealth endowment. $\theta_{i} \in T_{i}$ describes her preferences over bundles $\left(\omega, A_{i}\right)$ of an object $\omega \in \Omega$ and total wealth $A_{i} \in \mathbb{R}$. Preferences $\theta_{i}$ can be represented by a von NeumannMorgenstern utility function $u\left(\cdot, \cdot, \theta_{i}\right): \Omega \times \mathbb{R} \rightarrow \mathbb{R}$. $T_{i}$ denotes the set of all possible types $t_{i}$ for agent $i$ and $T=\left(T_{i}\right)_{i \in N}$ is the space of all type profiles $t=\left(t_{i}\right)_{i \in N}$. With $t_{-i}$ we refer to the type profile of all agents except agent $i$. In contrast to most mechanism design models we do not assume quasi-linear preferences but that wealth effects play a role. Particularly, the set of admitted types $T_{i}$ comprises all types $t_{i}=\left(\theta_{i}, e_{i}\right)$ with $e_{i} \in \mathbb{R}$ and $\theta_{i}$ such that $u\left(\cdot, \cdot, \theta_{i}\right)$ satisfies the following conditions.

- Monoton and continuous in wealth: For any $\omega \in \Omega, u\left(\omega, \cdot, \theta_{i}\right)$ is strictly monotonically increasing and continuous in total wealth $A_{i}$ 
- Strict and income independent object ranking: Preferences $\theta_{i}$ imply a strict and unique rank order of objects denoted by $r_{i}$, i.e. $u\left(\omega, A_{i}, \theta_{i}\right) \neq u\left(\omega^{\prime}, A_{i}, \theta_{i}\right) \Leftrightarrow$ $\omega \neq \omega^{\prime}$ and $u\left(\omega, A_{i}, \theta_{i}\right)>u\left(\omega^{\prime}, A_{i}, \theta_{i}\right) \Rightarrow u\left(\omega, A_{i}^{\prime}, \theta_{i}\right)>u\left(\omega^{\prime}, A_{i}^{\prime}, \theta_{i}\right)$ for all $A_{i}^{\prime} \in \mathbb{R} \cdot{ }^{10}$.

- Income effects: Let $\theta_{i}$ be such that object $\omega$ is preferred over $\omega^{\prime}$. For any $m>0$ there exist $\bar{A}_{i}>\underline{A}_{i}$ such that $u\left(\omega, A_{i}-m, \theta_{i}\right)>u\left(\omega^{\prime}, A_{i}, \theta_{i}\right)$ for all $A_{i}>\bar{A}_{i}$ and $u\left(\omega^{\prime}, A_{i}+m, \theta_{i}\right)>u\left(\omega, A_{i}, \theta_{i}\right)$ for all $A_{i}<\underline{A}_{i}$

The way income affects preferences is crucial for our analysis. We assume that wealth does not influence the ranking of objects of an agent (see second condition). However, wealth influences the willingness to pay and accept. The third condition implies that the willingness to pay for an object improvement becomes arbitrary high for increasing wealth while the willingness to accept becomes arbitrary low for decreasing wealth. An example for a utility function that satisfies the conditions above is $u\left(\omega, A_{i}, \theta_{i}\right)=v\left(\omega, \theta_{i}\right)+\left(1-e^{-\lambda\left(\theta_{i}\right) A_{i}}\right)$ where $v\left(\cdot, \theta_{i}\right)$ is any function that assigns a wealth independent value to each object. $\lambda\left(\theta_{i}\right)$ might depend on the type as well. ${ }^{11}$

We will be interested in shared characteristics of different types. For this we introduce equivalence classes for different characteristics.

$T_{i}\left(r_{i}\right)$ denotes the equivalence class of all types that describe the same ordinal ranking $r_{i}$ of objects. While all types in $T_{i}\left(r_{i}\right)$ agree on the ranking of object, they might disagree on what any object improvement is worth. This heterogeneity basically can have two reasons. First, even if $t_{i}$ and $t_{i}^{\prime}$ might have the same wealth level, they might differ in cardinal appreciation for the objects that is described by $\theta_{i}$ and $\theta_{i}^{\prime}$. Second, even if for $t_{i}$ and $t_{i}^{\prime}$ preferences are equal, i.e. $\theta_{i}=\theta_{i}^{\prime}, t_{i}$ might describe a wealthier agent than $t_{i}^{\prime}$ and thus this agent might be willing to pay more for an object improvement.

$T_{i}\left(\theta_{i}\right)$ denotes the equivalence class of all types that describe the same preferences $\theta_{i}$. Thus, all types in $T_{i}\left(\theta_{i}\right)$ do only differ according to their initial wealth endowment, i.e. for $t_{i}=\left(\theta_{i}, e_{i}\right)$ and $t_{i}^{\prime}=\left(\theta_{i}^{\prime}, e_{i}^{\prime}\right), t_{i}, t_{i}^{\prime} \in T_{i}\left(\theta_{i}\right)$ is equivalent to $\theta_{i}=\theta_{i}^{\prime}$. If two types $t_{i}$ and $t_{i}^{\prime}$ both belong to $T_{i}\left(\theta_{i}\right)$ they also belong to the same equivalence class of ranking $T_{i}\left(r_{i}\right)$. Thus, $T_{i}\left(\theta_{i}\right) \subset T_{i}\left(r_{i}\right)$. If two types $t_{i}$ and $t_{i}^{\prime}$ in $T_{i}\left(\theta_{i}\right)$ disagree on

\footnotetext{
${ }^{10}$ The assumption of unique rank orders technically already follows by the assumptions of continuity and strict rankings. However, due to its importance, we unhide it in this way.

${ }^{11}$ We allow for much broader class of utility function and, for instance, do not explicitly assume that the willingness to pay is strictly increasing in wealth. However, restricting the utility functions to, for instance, those of shape $u\left(\omega, A_{i}, \theta_{i}\right)=v\left(\omega, \theta_{i}\right)+\left(1-e^{-\lambda A_{i}}\right)$ does not change our analysis and results.
} 
what an object improvement is worth, this can only be due to heterogeneity in wealth levels.

$T_{i}\left(e_{i}\right)$ denotes the equivalence class of all types with equal wealth endowment $e_{i}$, i.e. for $t_{i}=\left(\theta_{i}, e_{i}\right)$ and $t_{i}^{\prime}=\left(\theta_{i}^{\prime}, e_{i}^{\prime}\right), t_{i}, t_{i}^{\prime} \in T_{i}\left(e_{i}\right)$ is equivalent to $e_{i}=e_{i}^{\prime}$.

We call an outcome of the problem an assignment $x=(\sigma, m) \in \mathcal{X} \subset \Omega^{n} \times \mathbb{R}^{n}$ that assigns exactly one object to each agent and determines monetary transfers. ${ }^{12}$ $\sigma \in \Omega^{n}$ describes the matching of objects to agents where $\sigma_{i}=\omega$ means that object $\omega$ is assigned to agent $i$. One object can at most be assigned to one agent, i.e. $\sigma_{i} \neq \sigma_{j}$ for $i \neq j . m \in \mathbb{R}^{n}$ describes the assignment of money and $m_{i} \in \mathbb{R}$ is the money agent $i$ receives or has to pay. $\mathcal{X}$ is the set of all outcomes. Each type $t_{i}$ uniquely defines preferences over outcomes. Particularly, agent $i$ of type $t_{i}=\left(\theta_{i}, e_{i}\right)$ evaluates her individual outcome $\left(\sigma_{i}, m_{i}\right)$ according to $u\left(\sigma_{i}, A_{i}, \theta_{i}\right)$ with $A_{i}=e_{i}+m_{i}$ being agent $i$ 's ex-post wealth. Because we do not consider quasi-linear preferences knowing $\theta_{i}$ is not sufficient to evaluate outcomes but we also need to know an agent's wealth endowment. Two agents with the same preferences $\theta_{i}$ over objects and total wealth might evaluate outcomes differently due to differences in wealth. On the other hand, two agents might evaluate outcomes in the same way but their types differ.

$\varphi=(\sigma, m)$ denotes a social choice function or direct mechanism (if types are private information) that selects for each type profile $t \in T$ an outcome $\varphi(t)=$ $(\sigma(t), m(t))$. We call $\sigma: \Theta \rightarrow M$ an object assignment (or matching) and $m: \Theta \rightarrow \mathbb{R}$ the money assignment (or transfers). ${ }^{13} \varphi$ might use tie-breaking rules like priorities (e.g. based on districts in school choice) and lotteries. We assume that those tie-breakers are determined before the mechanism and are fixed for each agent independent of the realization of types. Thus we concentrate on deterministic outcomes instead of lotteries over deterministic outcomes (we will later discuss how the results translate in case of probabilistic outcomes). In this sense, we do not restrict our considerations to anonymous mechanisms since agents might differ according to priorities or a lottery number.

We say that a social choice function $\varphi^{\prime}$ (or an object assignment $\sigma^{\prime}$ ) Paretodominates $\varphi($ or $\sigma$ ) if for all type profiles $t$ all agents are weakly better off and at least for one $t$ there is one agent that is strictly better off.

A social choice function $\varphi=(\sigma, m)$ is implementable if it it can be implemented as a dominant strategy equilibrium of a direct mechanism. By the revelation principle, for implementability we limit our attention to social choice functions where

\footnotetext{
${ }^{12}$ Particularly, we assume that no agent remains unassigned. The setting can be easily extended by adding an object $\emptyset$ with $n$ copies to $\Omega$ where $\emptyset$ corresponds to remaining unassigned.

${ }^{13}$ With a slight abuse of notation we denote by $\sigma$ the assignment that maps profiles to a matching as well as the matching selected. The same holds for $m$.
} 
truthtelling is a dominant strategy. Truthtelling is a dominant strategy if and only if $u_{i}\left(\sigma_{i}(t), A_{i}+m_{i}(t), \theta_{i}\right) \geq u_{i}\left(\sigma_{i}(t), A_{i}^{\prime}+m_{i}(t), \theta_{i}^{\prime}\right)$ for each agent $i$ and all $t_{i}, t_{i}^{\prime} \in T_{i}$ and $t_{-i} \in T_{-i}$.

According to Satterthwaite and Sonnenschein (1981) a social choice function $\varphi$ is non-bossy if for any agent $i \varphi_{i}\left(t_{i}, t_{-i}\right)=\varphi_{i}\left(t_{i}^{\prime}, t_{-i}\right)$ implies that for all agents $j$ $\varphi_{j}\left(t_{i}, t_{-i}\right)=\varphi_{j}\left(t_{i}^{\prime}, t_{-i}\right)$ holds. Thus, an agent cannot change another agent's assignment without changing her own. Non-bossiness of $\sigma$ and $m$ are defined analogously. ${ }^{14}$

Extensions. For simplicity we do not assume that objects have copies. The existence of copies does not change any of our analysis and results. In the basic model we do assume that agents might remains unassigned but that they do not have an outside option that they are free to choose. However, introducing an outside option (e.g. staying unassigned) allows for a straight-forward transfers of our definition of discrimination-freeness and therefore we discuss it later as an extension. The same holds for a two-sided market where the providers of the objects are strategic players as well and where for the providers there exists a concern for discrimination as well.

\section{Discrimination-Free Social Choice Functions}

In our model we refrain from the typical restriction of a matching market that monetary transfers are not allowed. Instead, in the following we introduce a desideratum based on moral concerns that appears to be a main root for repugnance towards monetary transfers: discrimination-freeness with respect to wealth. A social choice function is discrimination-free if an agent's object assignment does not depend on her wealth endowment. Hence, discrimination-freeness refers what determines how objects are allocated but does not a priori impose restrictions on transfers.

Definition 1 (Discrimination-Free). A social choice function $\varphi=(\sigma, m)$ is discrimination-free (with respect to wealth) if an agent's object assignment is independent of her wealth endowment, i.e. for any agent $i, \theta_{i}$ and $t_{-i}$ fixed

$$
\sigma_{i}\left(t_{i}, t_{-i}\right)=\sigma_{i}\left(t_{i}^{\prime}, t_{-i}\right) \text { for all } t_{i}, t_{i}^{\prime} \in T_{i}\left(\theta_{i}\right)
$$

$\varphi$ discriminates if it is not discrimination-free.

\footnotetext{
${ }^{14}$ Whether or not non-bossiness is a desirable characteristic of a social choice function appears to be disputable. Thomson (2014), for instance, discusses several interpretations of non-bossiness and questions their validity. However, we won't further elaborate on why non-bossiness might be required. Non-bossiness is not a critical requirement for our results and we rather discuss additional implications that result if non-bossiness is required.
} 
Agents that differ only in their wealth endowment but not in their preferences have to receive the same object via a discrimination-free social choice function. If we considered quasi-linear utilities, preferences over outcomes would not depend on wealth and with it discrimination-freeness would not impose restrictions on how a social choice function depends on preferences. However, as we assume income effects, discrimination might become a valid concern. For illustration consider two agents and two objects and assume that both agents like object $a$ more than object $b$. One agent is willing to pay more to receive object $a$ instead of object $b$ than the other one. The difference in the willingness to pay might solely come from a difference in wealth. A discrimination-free social choice function must not take account this difference. However, if the difference in the willingness to pay solely comes from a difference in preferences, a discrimination-free social choice function might regard it. Reconsidering the question of admitting trades among agents or not, requiring discrimination-freeness implies trading objects for money is allowed as long as the trade realizes for any wealth states of the agents.

An appealing feature of discrimination-freeness is that to judge whether or not this fairness criteria is satisfied it is sufficient to consider only one agent. This particularly allows us to concentrate on deterministic assignments. ${ }^{15}$

Discrimination-freeness does not impose any a-priori-restrictions on transfers but does limit to what extend a social choice function can exploit preferences information. So there appears to be a trade-off between discrimination-freeness and efficiency. In the following we discuss efficiency consequences of requiring discrimination-freeness. Furthermore, we discuss how discrimination-freeness restrict the set of implementable social choice functions.

\subsection{Efficiency}

Free markets allow to transfer utility via money and therefore offer the opportunity to realize Pareto-improvements via trades of objects and money. Markets without transfers provide less opportunities for Pareto-improvements. In any environment without transfers, ordinal mechanisms that assign objects to agents based on the agents' object rankings are at the efficient frontier of all admissible mechanisms. However, agents that agree on the object ranking might disagree on what an object improvement is worth. Generally, any ordinal mechanism without transfers can be

\footnotetext{
${ }^{15}$ By contrast, a popular fairness criteria in the literature of matching markets is equal treatment of equal (see Bogomolnaia and Moulin (2001), i.e. equal agents should receive the same consumption. Consumption then has to be random. Furthermore, in contrast to our setting, it is not always clear which agents should be considered as "equals".
} 
Pareto-improved by involving transfers.

In the following we show that money-free social choice functions cannot be Paretoimproved by involving transfers among agents without violating discrimination-freeness. The next proposition presents an even larger class of social choice functions (possibly involving transfers) that are inefficient but at the efficient frontier of discriminationfree social choice function. We call a social choice function efficient if it cannot be Pareto-improved within the same budget. We will show that efficiency and discrimination-freeneess do not generally exclude each other. However, efficiency and discrimination-freeness are only compatible if the social choice function is wealth adjusting. With wealth adjusting we precisely mean the following.

Definition 2 (Wealth-Adjusting). A social choice function $\varphi=(\sigma, m)$ is wealthadjusting if for any agent $i, \theta_{i}$ and $t_{-i}$ fixed one of the following conditions holds

- Agent $i$ 's ex-post wealth $A_{i}\left(e_{i}\right)=e_{i}+m_{i}\left(\theta_{i}, e_{i}, t_{-i}\right)$ in dependence of her own wealth endowment $e_{i}$ is bounded above or below, i.e. there exists $K \in \mathbb{R}$ with either $A_{i}\left(e_{i}\right)<K$ for all $e_{i} \in \mathbb{R}$ or $A_{i}\left(e_{i}\right)>K$ for all $e_{i} \in \mathbb{R}$.

- There is an agent $j \neq i$ whose ex-post wealth $A_{j}\left(e_{i}\right)=e_{j}+m_{j}\left(\theta_{i}, e_{i}, t_{-i}\right)$ in dependence of agent $i$ 's wealth endowment $e_{i}$ is not bounded, i.e. there does not exist $\underline{A}<\bar{A}$ such that $A_{j}\left(e_{i}\right) \in[\underline{A}, \bar{A}]$ for all $e_{i} \in \mathbb{R}$

Social choice functions that are not wealth adjusting neither cap one agent's wealth when she gains or looses wealth (first condition) nor they redistribute wealth in the sense, that there is an agent that becomes arbitrary wealthy or poor if another agent gains or looses wealth (second condition). Examples for social choice functions of further interest that do not adjust wealth are those that do not use any monetary transfers (i.e. $m=0$ ) and that have constant transfers (i.e. $m(t)=m\left(t^{\prime}\right)$ ). Social choice functions that adjust wealth are, for instance, those that adjust every agent's wealth to a constant, predefined wealth level or those that redistribute wealth in such a way that wealth is equalized among all agents.

Proposition 1. Consider a discrimination-free social choice function $\varphi=(\sigma, m)$ that is not wealth-adjusting. Assume that $\sigma$ is not Pareto-dominated by any other object assignment $\sigma^{\prime}$.

Any $\varphi^{\prime}$ with $\sum_{i} m_{i}^{\prime}=\sum_{i} m_{i}$ that Pareto-dominates $\varphi$ does discriminate. Furthermore, $\varphi$ is not efficient, i.e. there is a social choice function $\varphi^{\prime}$ with $\sum_{i} m_{i}^{\prime}=\sum_{i} m_{i}$ that Pareto-dominates $\varphi$.

Proof. See Appendix. 
Main driver of the proposition is how wealth affects preferences. Particularly, we exploit that the amount of money compensating an agent for receiving a worse object becomes arbitrarily large when wealth of this agent increases (since $\varphi$ is not wealth-adjusting). On the other hand, there is a maximal amount of money that each agent is willing to pay for a better object when fixing the initial level of wealth. This amount can be chosen independently of the other agent's wealth (again since $\varphi$ is not wealth-adjusting). This implies that if $\varphi^{\prime}$ is a Pareto-improvement of $\varphi$ as described in the proposition $\varphi^{\prime}$ has to discriminate. The reason is that an agent who received a worse object under $\varphi^{\prime}$ cannot be compensated any more for this object impairment when being wealthy enough. Therefore, if $\varphi^{\prime}$ Pareto-improves $\varphi$ her object assignment needs to change when gaining wealth. This implies that $\varphi^{\prime}$ is not discrimination-free. To show that $\varphi$ is not efficient we consider a type profile where all agents agree on the ranking. Just by changing one agent's wealth level we then can, by exploiting the non-linearity, induce a situation where a trade of objects among agents is a Pareto-improvement.

Proposition 1 implies that the efficiency limits that can be reached by only considering ordinal information and not using transfers, are already efficiency limits of discrimination-free social choice functions. Any ordinal object assignment $\sigma$ that is not Pareto-dominated by any other ordinal object assignment $\sigma^{\prime}$ is also not Paretodominated by any object assignment that is possibly not ordinal but might depend on the full type profile. By Proposition 1, a social choice function $\varphi=(\sigma, 0)$ that does not use transfers (and which is therefore not wealth-adjusting) then cannot be Pareto-dominated without violating discrimination-freeness. This is summarized by the corollary below.

Corollary 1. Consider any ordinal object assignment $\sigma$, i.e. for any ranking $r_{i}$ of objects $\sigma\left(t_{i}, t_{-i}\right)=\sigma\left(t_{i}^{\prime}, t_{-i}\right)$ holds for all $t_{i}, t_{i}^{\prime} \in T_{i}\left(r_{i}\right)$ and $t_{-i} \in T_{-i}$.

If $\sigma$ is not Pareto-dominated by any other ordinal object assignment $\sigma^{\prime}$ then $\varphi(t)=$ $(\sigma(t), 0)$ is at the efficient frontier of all budget-balanced discrimination-free social choice functions.

A second direct implication of Proposition 1 is that if any social choice function is efficient and discrimination-free it has to adjust wealth levels. In the following we furthermore show that efficiency and discrimination-freeness are not exclusive.

Corollary 2. There is a discrimination-free social choice function $\varphi=(\sigma, m)$ that is efficient, i.e. no social choice function $\varphi^{\prime}=\left(\sigma^{\prime}, m^{\prime}\right)$ with the same budget $\sum_{i} m_{i}^{\prime}(t)=$ $\sum_{i} m_{i}(t)$ for all $t \in T$ that Pareto-dominates $\varphi$ exists. Any social choice function that is discrimination-free and efficient is wealth-adjusting. 
An example for a discrimination-free and efficient social choice function is the following that performs the assignment in two steps. First, wealth of all agent is adjusted according to a predefined wealth level which is independent of the initial wealth endowment $e_{i}$. For instance, think of a mechanism that first assures that every agent faces a wealth level $A>0$. Second, given this new income distribution the mechanism assigns objects such that utilities are maximized. This allocation is efficient. Furthermore, an agent's object assignment is independent of her wealth endowment. $\varphi$ is wealth-adjusting because each agent's ex-post wealth does not depend on her initial wealth endowment. The social choice function described is not necessarily budget balanced. However, if wealth endowments are drawn from a distribution such that expected total endowment is $\mathbb{A}$, the mechanism above is budget balanced in expectation if wealth is adjusted in such a way that the sum of wealth equals $\mathbb{A}$. It is even feasible to construct a mechanism that redistributes wealth such that it is efficient and ex-post budget balanced. ${ }^{16}$

Merely the fact that the introduction of money cannot be used to yield efficiency gains compared to assignments without transfers does not necessary imply that there is no use for money at all. Money might be used for funding resources. Roots for heterogeneity in payments then might be heterogeneity in wealth endowments or heterogeneity in assigned resources (e.g. due to distributive concerns). Furthermore, we saw that discrimination-free assignments without transfers are not efficient while efficient and discrimination-free social choice functions with transfers exists. We did not yet account for issues of implementability. Once types are not public information implementability issues further restrict the design of payments as discussed in the next subsection.

Ex-ante improvements. Proposition 1 implies that exploiting any information about preferences beyond object rankings does not yield Pareto-improvements compared to a money-free assignment. In our analysis we concentrate on deterministic assignments and therefore take an ex-post perspective. If we allowed for probabilistic outcomes cardinal information might be exploited by Pareto-improving ex-ante welfare via conducting lotteries. With some further specifications of preferences, we can transfer Proposition 1 to probabilistic outcomes by replacing Pareto-dominance by ex-ante Pareto-dominance. Then, if $\sigma$ is not ex-ante Pareto-dominated by any discrimination-free matching $\sigma^{\prime}$ there is no discrimination-free social choice function

\footnotetext{
${ }^{16}$ Such a mechanism can be constructed by allocating objects such that for any specific wealth endowment no Pareto-improvements are feasible via transfers. Then, for any other wealth endowment it is possible to redistribute wealth such that this allocation of object cannot be Pareto-improved within the budget.
} 
with $\sum_{i \in N} m_{i}=0$ that ex-ante Pareto-dominates $\varphi \cdot{ }^{17}$ In this case information about preference intensities might be exploited to gain efficiency. Thus, lotteries can only ex-ante Pareto-improve pure matchings without violating discrimination-freeness if the preferences over the lotteries are not wealth dependent. Wealth does not influence rank order list but it might influence cardinalities. For instance, the poorer an agent is the more risk averse she might be.

\subsection{Implementability}

If types are private information we are not only interested in what social choice functions are desirable but also what social choice functions are implementable. In a setting of incomplete information we consider direct mechanisms that select an outcome based on agents' type reports. Requiring implementability then further restricts the admissible type dependence of the mechanisms is restricted.

We first assume that no information about the type is available, i.e. both the preference profile $\theta=\left(\theta_{i}\right)_{i \in N}$ and the wealth profile $e=\left(e_{i}\right)_{i \in N}$. Later we consider the case that information about the wealth profile $e=\left(e_{i}\right)_{i \in N}$ is available. Both cases provide a foundation for assigning objects without using transfers and payments independent of preferences (e.g. object-independent lump-sums or taxes), as the next results show.

Proposition 2. Let $\varphi=(\sigma, m)$ be an implementable social choice function. $\varphi$ is discrimination-free if and only if for each agent $i$ and $t_{-i}$ fixed, agent $i$ 's

- Money assignment $m_{i}\left(\cdot, t_{-i}\right)$ is independent of her type, i.e. $m_{i}\left(t_{i}, t_{-i}\right)=m_{i}\left(t_{i}^{\prime}, t_{-i}\right)$ for all $t_{i}, t_{i}^{\prime} \in T_{i}$.

- Object assignment $\sigma_{i}\left(\cdot, t_{-i}\right)$ only depends on her ranking, i.e. for all $r_{i}$ $\sigma_{i}\left(t_{i}, t_{-i}\right)=\sigma_{i}\left(t_{i}^{\prime}, t_{-i}\right)$ for all $t_{i}, t_{i}^{\prime} \in T_{i}\left(r_{i}\right)$.

If $\sigma$ and $m$ are additionally non-bossy, $\varphi$ is discrimination-free if and only if $m$ is independent of $t=\left(t_{i}\right)_{i \in N}$ and $\sigma$ only depends on the agents' rank order lists $r=\left(r_{i}\right)_{i \in N}$.

Proof. See Appendix.

To get the intuition for the proof first note that discrimination-freeness and implementability of a social choice function imply that not only the object assignment but also the money assignment is independent of wealth. Then assume that the monetary payments are not type independent, i.e. there are types $t_{i}$ and $t_{i}^{\prime}$ such that

\footnotetext{
${ }^{17}$ Details available upon request.
} 
$m_{i}\left(t_{i}\right)<m_{i}\left(t_{i}^{\prime}\right)$. Any agent $i$ with the same preferences as $t_{i}$ but a different wealth level is assigned to the same object (due to discrimination-freeness) and the same transfers (due to implementability). However, if an agent of type $t_{i}$ looses enough wealth she prefers to report $t_{i}^{\prime}$ instead of her true type. This then contradicts implementability. The restriction on $\sigma$, i.e. $\sigma_{i}$ must only depend on agent $i$ 's ordinal ranking, is implied by the restrictions on $m$ : Since $m_{i}$ is independent of agent $i$ 's type, considering more information than rank order lists into account for the object allocation contradicts implementability.

Proposition 2 shows that to implement a discrimination-free social choice function the report of the agents can be restricted to rank order lists. Any discrimination-free and implementable social choice functions then has the form

$$
\varphi\left(\theta_{i}, e_{i}\right)=\left(\sigma_{i}\left(r_{i}\right), m_{i}\right) \text { for } \theta_{i} \in T_{i}\left(r_{i}\right) \text { and } m_{i} \in \mathbb{R} \text { constant. }
$$

$\varphi$ thus can be decomposed into a classical matching problem that depends on rank order lists and constant (type independent) payments. No information that goes beyond ordinal rankings can be exploited by an implementable and discriminationfree social choice function.

By combining the results of Proposition 1 and Proposition 2, ordinal matchings with constant transfers (e.g. no transfers) are already at the efficient frontier of discrimination-free social choice function if the matching is not Pareto-dominated by any other matching without transfers. Furthermore, ordinal matchings with constant transfers are the only social choice functions that we might reach at the efficient frontier of discrimination-free social choice functions if implementability is required.

We indeed can implement social choice functions at the efficient frontier, for instance via Serial-Dictatorship. ${ }^{18}$ Inefficiencies of such a mechanism without transfers are obtained as second-best outcomes when requiring discrimination-freeness. In the context of school choice problems where students might be ordered according to a priority structure other popular ordinal matchings where truthtelling is a dominant strategy are the Deferred-Acceptance-Algorithm proposed by Gale and Shapley (1962) or the Top Trading Cycles Mechanisms (see, for instance, Abdulkadiroğlu and Sönmez (2003)). The last mentioned is at the efficient boarder of transfer-free assignment while the first one is not.

Availability of wealth information. Proposition 2 deals with a setting where the whole type is unknown. We now asses a setting where wealth endowments are known

\footnotetext{
${ }^{18}$ As we concentrate on deterministic matchings, any lotteries that might be needed for serial dictatorship (or other mechanisms) are assumed to be conducted before the matching takes place.
} 
while preferences are still not.

Proposition 3. Let $\varphi=(\sigma, m)$ be an implementable social choice function. Assume that for any agent $i, \theta_{i}$ and $t_{-i}$ fixed, agent $i$ 's ex-post wealth $A_{i}=e_{i}+m_{i}\left(\theta_{i}, e_{i}, t_{-i}\right)$ is not constant in her wealth endowment $e_{i} . \varphi$ is discrimination-free if and only if for every agent $i$ and $t_{-i}$ fixed, agent $i$ 's

- Money assignment $m_{i}\left(\cdot, t_{-i}\right)$ is independent of her preferences, i.e. for all $e_{i}$ $m_{i}\left(t_{i}, t_{-i}\right)=m_{i}\left(t_{i}^{\prime}, t_{-i}\right)$ for all $t_{i}, t_{i}^{\prime} \in T_{i}\left(e_{i}\right)$

- Object assignment $\sigma_{i}\left(\cdot, t_{-i}\right)$ only depends her object ranking, i.e. for all $r_{i}$ $\sigma_{i}\left(t_{i}, t_{-i}\right)=\sigma_{i}\left(t_{i}^{\prime}, t_{-i}\right)$ for all $t_{i}, t_{i}^{\prime} \in T_{i}\left(r_{i}\right)$

If $\sigma$ and $m$ are non-bossy, $\varphi$ is discrimination-free if and only if $m$ is independent of $\theta=\left(\theta_{i}\right)_{i \in N}$ and $\sigma$ only depends on the agents' rank order lists $r=\left(r_{i}\right)_{i \in N}$.

Proof. See Appendix.

Analogously to Proposition 2, a discrimination-free social choice function with non-constant ex-post wealth can be decomposed into a classical matching based on rank order lists and payments that are not linked to the preferences. Discriminationfree and implementable social choice functions have the form

$$
\varphi\left(\theta_{i}, e_{i}\right)=\left(\sigma_{i}\left(r_{i}\right), m_{i}\left(e_{i}\right)\right) \quad \text { for } \quad \theta_{i} \in T_{i}\left(r_{i}\right)
$$

In real market applications $A_{i}$ might not be fully known, but wealth related information like the yearly income, assets etc. might be available. Payments then continue to be independent of preferences and might only depend on the information available. Thus, Proposition 3 can be interpreted as: Even if full information about wealth was available, payments need to be independent of preferences.

To prove the proposition we again construct a type that has an incentive to misreport if transfers are not constant. However, it is slightly more complex to construct this type compared to Proposition 2. While for Proposition 2 it was sufficient to vary one agent's wealth, this proof uses constructions of preferences such that the object allocation under $\varphi$ differs for different levels of wealth. If ex-post wealth is not independent of $e_{i}$ that choice sets are indeed different for two wealth endowments $e_{i}$ and $e_{i}^{\prime} \cdot{ }^{19}$ The choice set is the set of available outcomes (here defined as bundles of object and total ex-post wealth) that an agent of a certain level of initial wealth has by varying the report.

\footnotetext{
${ }^{19}$ For instance, any mechanisms that first assures that each agent has a predefined level of financial wealth and then applies a market solution is discrimination-free as a wealth change of any agent does not change the final object allocation nor the ex-post wealth.
} 
The only exception that allows a discrimination-free mechanism to exploit information about preferences beyond rankings is if ex-post wealth is made constant with respect to wealth endowments. Proposition 3 implies that if $\varphi$ depends on more information about the preferences than only rank order lists, ex-post wealth has to be independent of initial wealth. As an example consider the mechanism presented in the context of Corollary 2. First, the mechanism adjusts each agent's wealth level to any predefined wealth level that is independent of her initial wealth (possibly the same for all agents). Then, the mechanism assigns objects and money. In this second step of the mechanisms, preferences $\theta=\left(\theta_{i}\right)_{i \in I}$ can play a role but not wealth endowments $e=\left(e_{i}\right)_{i \in I}$. Particularly, if we consider the wealth levels that result after the first step and the preferences of the agents as the new types, the mechanisms in the second step have all the freedom that mechanisms have where we do not impose discrimination-freeness.

\subsection{Extensions}

Outside options. In many real markets outside options are available. This is, for instance, the case if a private market co-exists. We can integrate an outside option into our model via adding an outcome $\left(\omega_{o}, m_{o}\right)$ with $n$ copies to $\Omega$. An agent can freely choose the outcome $\left(\omega_{o}, m_{o}\right)$. Hence, we consider only social choice functions that assign for any type profile and any agent nothing worse than $\left(\omega_{o}, m_{o}\right)$. This further restricts the implementable social choice functions that are discriminationfree. First, if $\varphi$ is implementable and discrimination-free each agent is assigned to an object that is at least as good as the object of the outside option $\omega_{o}$. Otherwise agents that are rich enough would choose the outside option. Second, any transfers have to be greater or equal to $m_{o}$. Otherwise agents that are poor enough prefer the outside option to their assignment.

Two-sided matching. We considered a one-sided model in the sense that only agents on one side of the market are strategic players in the game and only the agents' welfare is of interest. Providers of objects might be active market participants and strategic players as well. Discrimination-freeness then might be required for both sides of the markets. Consequential restrictions follow analogously for each side of the market.

Seller and buyer model. A special case is a setting where a seller is willing to sell an object for a certain price and a buyer is willing to buy it for a certain price. If the seller's will-sell-price is lower than the buyer's will-buy-price the transaction takes place. Discrimination-freeness then translates to whether or not the trade takes place 
must not depend on financial wealth of both the seller and the buyer. Considering a predetermined and fixed price, our specification of preferences implies that to ensure discrimination-freeness the price has to be zero. For any fixed price that is not zero there are wealth levels such that the transaction takes place and for others not.

Modifying income effects. Our model assumes that preferences are such that the willingness to pay for object improvements and the willingness to accept for object impairments become arbitrary low when an agent looses wealth. In the following we discuss how a modification of this assumption affects our results.

First, we consider the case that each agent has a minimum willingness to pay $\underline{m}>0$ for an object improvement, i.e. each agent prefers a better object without transfers to a worse object with a transfer smaller than $\underline{m}$. Then ordinal object assignments without transfers are still at the efficient frontier of discrimination-free social choice functions. This is because a compensation for an object impairment at a certain level of initial wealth is still not sufficient for higher levels of financial wealth. Propositions 2 and 3 in a setting of incomplete information slightly change. Payments might depend on type - but differences in payments are bounded above by $\underline{m}$. The object allocation still must only depend on ordinal preferences.

Second, assume that wealth endowments are bounded below, i.e. $e_{i} \in\left[\underline{e}_{i}, \infty\right)$. Implications are similar to those of a minimum willingness to pay. For each possible $\theta_{i}$ there exists $\underline{m}_{i}$ such for all wealth levels $e_{i} \in\left[\underline{e}_{i}, \infty\right)$ an agent of type $t_{i}=\left(\theta_{i}, e_{i}\right)$ is at least willing to pay $\underline{m_{i}}$ for an object improvement. By the same argument as above, ordinal object assignments without transfers are still at the efficient frontier of discrimination-free social choice functions. If $\inf _{\theta_{i}} \underline{m}_{i}>0$ Proposition 2 and Proposition 3 change in exactly the same way as for the minimum willingness to pay. However, if $\inf _{\theta_{i}} \bar{m}_{i}=0$ for all agents $i$, Proposition 2 and Proposition 3 remain unchanged as only constant payments are eligible.

If wealth is also bounded above, i.e. $e_{i} \in\left[\underline{e}_{i}, \bar{e}_{i}\right]$ it might be the case (if $\bar{e}_{i}$ is small enough) that there exists $\bar{m}$ that always compensates for a worse object. Then Proposition 1 changes as here might be room for Pareto-improvements by using monetary transfers without violating discrimination-freeness. Though, for each Pareto-improvement it has to be ensured that the Pareto-improvement persists by any changes in wealth. Under incomplete information, the payments either have to be very small (see above) or very large, such that any wealth level would rather take them if $\inf _{\theta_{i}} \underline{m}_{i}>0$ and $\sup _{\theta_{i}} \bar{m}_{i}<\infty$.

The considerations above imply that more equal societies have more leeway in reaching efficient outcomes than unequal societies. An extreme case is that wealth is equally spread among a population. Then, discrimination-freeness is anyway ensured 
and the design of a social choice function is not restricted. Note that the arguments for bounded wealth are the same as seen in the the subsection on efficiency when discussing wealth-adjusting social choice functions.

Non-constant ranking. One main assumption on preferences of the agents is that the ranking of objects is wealth independent. Technically, the assumptions of continuity and strict preferences over objects imply constant rankings. Relaxing the assumption of continuity and requiring only continuity from below, ranking of objects might differ with wealth. For instance, wealthier agents might have another first choice than poorer agents. A priori it is not clear how to define discriminationfreeness when rankings change with wealth. Sticking to our definition would mean that even rankings must not play a role for the object distribution. An alternative is to treat agent's preferences as if the ranking was wealth-independent. This might be a valid approach if payments in the mechanism are small enough such that constant rankings are a reasonable approximation. However, then concerns for segregation rather than concerns for discrimination might become relevant.

\subsection{Examples and Applications}

If we refrain from considering mechanisms that assure constant ex-post wealth, Proposition 2 (the whole type is unknown) and Proposition 3 (wealth is known, preferences are not) imply that for incomplete information transfers have to be preferenceindependent and the object assignment must only depend on the ordinal ranking of objects. Applied to real markets this justifies the use of an ordinal matching mechanism possibly combined with payment to fund resources that is not linked to the outcome of the matching. In the following we discuss some mechanisms on real markets in the context of discrimination concerns.

School Choice. There exist various applications in school choice that use a centralized assignment procedure without monetary transfers. School places (at least at public schools) are often fully funded by taxes and parents do not have to pay additional fees. Furthermore, schooling (up to a certain age) is compulsory in most countries. Such school assignment procedures are then discrimination-free. However, this only holds as long as no private sector co-exists where fees are charged.

Compensation for kidney donors. The buyer and seller model discussed in the extension is especially interesting in the context of compensation for kidney donors. To increase donations several models of compensating donors are currently discussed intensively. Our model implies that a free market for kidney leads to discrimination. Furthermore, any lump-sum as a compensation for the donor leads to discrimination. 
In-kind incentives, on the other hand, do not lead to discrimination as long as they incentivize a donation independent on the wealth level of a person. However, our results also imply that the recent practice is not discrimination-free since donors must not receive any money. This is because donors have expenditures when donating a kidney. Then no reimbursement of costs leads to discrimination as wealthier people are rather willing to bear the costs. Gill et al. (2014), for instance show, that in the US the wealthier donate at a higher rate. Reimbursement of costs therefore would rather decrease discrimination than increase.

(Compulsory) health insurance. Health insurance systems in countries with compulsory insurance are often (roughly speaking) designed such that the payment for health insurance is a certain percentage of income while health insurers must not discriminate with respect to income. In the US, with Medicare there is made an effort to ensure discrimination-free insurance for people age 65 or older by making everybody eligible for Medicare. In Germany, health insurance is compulsory for all ages and payments for statutory health insurance are a fixed percentage of income. ${ }^{20}$ People have free choice of the health insurance provider as health insurers in general must not refuse insurees. This ensures that payments are preference-independent and the allocation is wealth independent. By imposing mandatory insurance and free choice a selection of the health insurer by quality-attributes rather than incomes is enforced. ${ }^{21}$ Fees ensure funding, difference in fees have solely distributive reasons. However, with a co-existing private market, discrimination occurs. In Germany, there are indeed ongoing complaints about a two-tier health care system as people above a certain income threshold are free to choose a private insurer. If private insurance does not only mean more comfort but even better health treatment, discrimination occurs. An example of a country where basically no private health insurance market co-exists is Austria.

Childcare. The assignment of childcare places in Germany is an example for a system that is partly tax-funded but also charges additional income-dependent fees. Local authorities themselves can decide on the concrete market design. Most German cities installed a system where parents pay an income dependent fee which is independent on the specific childcare center chosen. All costs exceeding this fee are funded by the local authority (i.e. via taxes). The specific assignment to the childcare centers then is executed separately from the transfers. Some cities use a decentralized system where parents directly apply at the childcare centers others use a centralized

\footnotetext{
${ }^{20}$ To be precise, the fees might vary slightly from insurer to insurer which again might lead to discrimination. As differences are rather small we won't consider here.

${ }^{21}$ The free choice people have when deciding for an insurer will be of special interest in the next chapter as it serves as an example for externality-free mechanisms.
} 
assignment where parents can submit preferences. Childcare centers do not have an incentive to select parents by income as they receive a lump-sum per child from the local authority.

Participation is not mandatory and therefore parents might decide based on their financial wealth whether or not to apply for a childcare place. In many cities parents with low income do not have to pay anything for childcare which at least ensures that fees do not prevent the poor from participating. However, if poorer parents send their child to childcare while wealthier parents do not, discrimination might be an issue.

Matching with "virtual money". Virtual money that has no outside use might be used instead of real money to allocate objects: Each agent receives a fixed amount of virtual money that she can split among several objects. Based on this, the allocation is made (e.g. via a Pseudomarket for probability shares as described in Hylland and Zeckhauser (1979)). Those mechanism are in the spirit of Proposition 2 if we allow for probabilistic outcomes (see also the discussion of Proposition 1 for probabilistic outcomes). Using real money to buy probability shares would lead to discrimination. Using faked money implies constant monetary transfers of zero. The only reason for the use of faked money is to elicit cardinal information. Therefore, discriminationfreeness can only be assured if those cardinal preferences are wealth independent.

\section{Use of Money Outside a Mechanism}

Even object assignments without transfers might result in discrimination. This is a valid concern as soon as there are ways to use money outside a market designer's control to change parameters of the game. Access to the goods then might depend on wealth and therefore discrimination-freeness is no longer assured. Various ways to influence an assignment outside a market designer's control might exist dependent on the parameters that determine outcomes of a mechanism. An example are neighborhood priorities in school choice: Moving houses to an area of a preferred school raises the chances to receive a seat at this school. Those being able to afford high housing prices have the choice where to live what in turn influences the access to schools. (Black, 1999) analyzed housing prices and showed that school quality is correlated with house prices. In the context of organ donations money might be used to gain priority as well. Steve Jobs, for instance, reportedly obtained his liver transplantation because he was advised to raise his changes by subscribing to waiting lists in other states than his home state California. ${ }^{22}$ This approach required to be

\footnotetext{
${ }^{22}$ See for instance http://edition.cnn.com/2009/HEALTH/06/24/liver.transplant.priority.lists/.
} 
rich enough to quickly be at any location. Co-existing private markets as discussed in the previous section are also examples where being wealthy results in access to better goods like private schools or private insurances.

In the following we consider a setting of incomplete information and model the use of money outside a mechanisms by bribes in the sense that one agent might bribe another agent to report false preferences. This includes as an example the case that somebody bribes somebody else to donate a kidney. Also, the examples mentioned above then can be interpreted as special cases of bribing if interpreting the corrupted parameters as preferences of an additional side of the market.

We first define what we mean by bribing in the spirit of Schummer (2000). We then discuss necessary and sufficient conditions for social choice functions that preserve discrimination-freeness under bribes. With discrimination-freeness under bribes we mean that even if one agent might bribe another agent the resulting outcome of a mechanism that implements this social choice function is discrimination-free. In the remainder of this section we discuss applications and how to transfer the results to other cases of using money outside the mechanism.

Definition 3 (Bribing). Let $\varphi=(\sigma, m)$ be a social choice function. Agent $i$ has an incentive to bribe agent $j$ if there is a profile $t=\left(t_{i}\right)_{i \in N}$, a corrupted type $t_{j}^{\prime} \neq t_{j}$ and a bribe amount $\tau \geq 0$ such that

- $u\left(\sigma_{i}\left(t_{j}^{\prime}, t_{-j}\right), e_{i}+m_{i}\left(t_{j}^{\prime}, t_{-j}\right)-\tau, \theta_{i}\right)>u\left(\sigma_{i}(t), e_{i}+m_{i}(t), \theta_{i}\right)$ and

- $u\left(\sigma_{j}\left(t_{j}^{\prime}, t_{-j}\right), e_{j}+m_{j}\left(t_{j}^{\prime}, t_{-j}\right)+\tau, \theta_{j}\right)>u\left(\sigma_{j}(t), e_{j}+m_{j}(t), \theta_{j}\right)$

$\varphi$ is bribe-proof if no incentives to bribe exist. For any social choice function $\varphi$ define $B(\varphi)$ as

$$
B(\varphi)=\left\{\varphi^{B_{i j}} \mid \varphi^{B_{i j}}(t)=\varphi\left(t_{j}^{B}, t_{-j}\right) \text { for all } t \in T\right\}
$$

where $t_{j}^{B}$ is a corrupted type of agent $j$ that is bribed by another agent $i$ who has an incentive to bribe. If no bribing incentives exist, $t_{j}^{B}=t_{j}$.

An agent therefore has an incentive to bribe another agent if paying another agent to state false preferences makes both agents better off. $B(\varphi)$ can be interpreted as the set of all possible assignments that might result by $\varphi$ if one agent bribes another agent. Particularly, if $\varphi$ is bribe-proof $B(\varphi)=\varphi$.

\subsection{Preserving Discrimination-Freeness Under Bribes}

In the following we extend the definition of discrimination-freeness to the case that bribes might occur. Roughly speaking we require that the outcomes of a social 
choice function if bribes might occur are discrimination-free. Our following definition of preserving discrimination-freeness under bribes concentrates on bribes that involve only two agents. The reason behind is that bribing requires a considerable amount of coordination among the agents. Thus, considering larger groups of agents involved in bribes makes bribes even less realizable. Our results can then be interpreted as even if we only consider situations where two agents are involved in bribing it implies the presented strict restrictions on social choice functions.

Definition 4 (Preserving Discrimination-Freeness Under Bribes). An implementable and discrimination-free social choice function preserves discrimination-freeness under bribes if all $\varphi^{B_{i j}} \in B(\varphi)$ are discrimination-free.

We are primarily interested in necessary and sufficient conditions such that an implementable and discrimination-free social choice function preserves discriminationfreeness under bribes. Obviously, a sufficient condition for preserving discriminationfreeness under bribes is bribe-proofness. We show that for discrimination-free social and implementable choice functions bribe-proofness is equivalent to externalityfreeness, i.e. an agent's outcomes does not depend on other agent's preferences. Nonbossiness of the social choice function makes bribe-proofness a necessary condition for preservation of discrimination-freeness under bribes.

Proposition 4. Let $\varphi$ be an implementable and discrimination-free social choice function. $\varphi$ is bribe-proof if and only if $\varphi$ is externality-free, i.e. $\varphi_{i}\left(t_{i}, t_{-i}\right)=$ $\varphi_{i}\left(t_{i}, t_{-i}^{\prime}\right)$ for all $t=\left(t_{i}\right)_{i \in N}$ and $t_{-i}^{\prime}$.

If $\varphi$ is non-bossy $\varphi$ preserves discrimination-freeness under bribes if and only if $\varphi$ is externality-free.

Proof. See Appendix.

The main intuition for the equivalence of bribe-proofness and externality-freeness is that once an agent can influence another agent's assignment, we can find an agent that is willing to pay a certain amount to profit from this difference while on the other hand, there is an agent that would be willing to accept this amount to misreport in favor of the first agent. To construct those types described we exploit that the social choice function is implementable and discrimination-free and that therefore one's payments are independent of one's type (see Proposition 2). ${ }^{23}$

\footnotetext{
${ }^{23}$ Bribe-proofness and externality-freeness can also be shown to be equivalent if we do not concentrate on discrimination-free social choice function. Schummer (2000), for instance, showed for the case of quasi-linear utility function with compensable preferences that bribe-proofness implies externality-freeness. This can be transferred to our preference characteristics.
} 
Bribe-proofness can become a necessary condition for preserving discriminationfreeness if whether bribing incentives exist or not depends on the wealth of the agents. This is particularly the case if the social choice function is non-bossy. Once a bribing incentive exists, agents poor enough can not anymore afford the bribe amount that is necessary to corrupt preferences of somebody else. Non-bossiness here ensures that the bribe amount that is necessary to bribe is not arbitrary small. For social choice functions that are not non-bossy, bribes might be quasi-free at a bossy point which makes bribes affordable for all wealth levels.

Our results show that the restrictions derived in the previous chapter are not sufficient any more if bribes might occur. Particularly, even object assignments without transfers might discriminate. Externality-freeness goes on cost of efficiency. If more objects than agents exist externality-freeness implies wastefulness, i.e. there exist a type profile such that there remains an object unassigned that is preferred by at least one agent to her assigned object.

Corollary 3. Let $\varphi$ be an implementable and non-bossy social choice function that is discrimination-free. Assume that more objects than agents are available, i.e. $n<k$. If $\varphi$ preserves discrimination-freeness under bribes then $\varphi$ is wasteful.

The corollary directly follows by Proposition 4. $\varphi$ needs to be externality-free which implies that the agents' choice are independent of preferences and pairwise disjoint. $n<k$ implies that for any type profile $t$ there exists an object $\omega$ that remains unassigned. If $\omega$ belongs to nobody's choice set it remains unassigned for all type profiles, even if somebody ranks $\omega$ first. Therefore, $\varphi$ is wasteful. If $\omega$ belongs to the choice set of some agent $i$ it does not belong to any other agent's choice set. Particularly, if any agent $j$ other than agent $i$ ranks $\omega$ first and all other types remain unchanged, object $\omega$ is still unassigned. Therefore, $\varphi$ is wasteful.

We considered one-sided markets. For two-sided markets the transfer of the results is analogously to the transfer described in the previous section. If agents might bribe providers (or even the other way around) to avoid discrimination-freeness costly bribes have to be avoided. For this, an agent's outcome has to be independent of preferences of the other side of the market. If parameters as presented in the introduction of this section are interpreted as substitutes for preferences of a second side of the market (like neighborhood-priority, paying a fee for a private school or subscribing on multiple waiting lists) an agent's outcome has to be independent of corruptible parameters to ensure discrimination-freeness. 


\subsection{On (Non-)Admissible Mechanisms and Applications}

Structure of externality-free mechanisms. Externality-free mechanisms can be described by considering the agents' choice sets - which are the allocations she can achieve via her reports given a report of the other agents. Externality-freeness then is equivalent to the choice set of an agent being constant. Therefore, the choice sets have to be build in such a way that they are disjoint, i.e. each object can only appear in one choice set. This implies how to build externality-free object allocations. First, independently of the types, $n$ disjoint subsets of $\Omega$ are build (e.g. via a lottery). Again, independently of the types, the subsets are assigned to the agents. Then $\sigma$ chooses for each agent an object out of the subset that is assigned to this particular agent. This is the only step where type-dependence is allowed. Special cases are the following. If there are exactly as many objects as agents, i.e. $k=n$, the allocation is constant, i.e., the allocation is completely type-independent. A simple lottery of the objects fulfills this condition. When copies are available, and assuming each object has at least $n$ copies, each agent can be provided with a choice set containing all objects and therefore always can receive her first choice.

Possibilities to improve assignments are limited if externality-freeness is desired. Levers are how to build the subsets or the amount of objects available. The following corollary describes how to best build the agents' choice sets and shows that expected utility of each agent is a concave function in the number of objects available. Hence, given a uniform distribution of types with regard to the valuation of objects, highest expected total welfare is obtained if choice sets of preferably equal size are build. This is because an agent that is facing a choice set of size $j$ randomly chosen out of a set $\Omega$ is facing decreasing utility gains when increasing the size $j$ of the set.

Corollary 4. Assume that the agents are homogeneous in the sense that their types are drawn from the same distribution. If every agent receives a random choice set of $\Omega$, the highest expected utility is achieved in case that the differences in size of the disjoint choice sets are minimal. Furthermore, total expected utility gains are decreasing with an increase in the number of objects.

Proof. See appendix.

Externality-free mechanisms appear to be relevant in many markets - various currently used mechanisms work in such a way that one person's assignment is independent of other person's preferences. In the following we discuss two examples in the context of externality-freeness.

Kidney transplants. It is current policy in many countries that the allocation of kidneys out of the cadaver queue to patients does not depend on the subjective pref- 
erences of the patients. Kidneys available are distributed based on exogenous factors such as urgency, region, blood type etc. (priority based matching) that makes manipulation very difficult. However, as soon as the report of those exogenous factors can be manipulated, there is room for discrimination as misreports could be incentivized by bribes. This is not just a theoretical case as a 2013 uncovered scandal in Germany regarding transplant corruption shows: doctors manipulated factors that determine priorities for receiving a kidney ${ }^{24}$. When considering living donations, we face a two-sided market where the preferences of the providers play a role. If anyone can donate a kidney to anyone needing it there is room for bribes and therefore for discrimination.

Health insurance. In Germany, roughly speaking, every citizen with earnings below a certain income threshold has to be insured by a statutory health insurance. Since 1996, everybody has the right to select their preferred (statutory) health insurer and the respective insurer has to accept everybody applying as an insurant. ${ }^{25}$ Thus, a first-choice allocation regarding the insurant's preferences is chosen (capacity of the health insurer is extended if needed). Before the law for ensuring free choice was introduced, the other extreme type of an externality-free mechanism was used. The insurant could not choose the health insurer but was assigned to an insurer depending on his or her occupation. In both cases neither other insurants' preferences nor the preferences of the health insurer itself play a role.

\section{Conclusion}

We analyze a setting in which a set of scarce and indivisible goods has to be assigned to agents. Our analysis shows that if society desires that access to resources is independent of financial wealth, money cannot be used to achieve efficiency gains in comparison to a pure distribution of the goods without transfers. Efficiency can only be achieved without violating discrimination-freeness if wealth levels are adjusted by the mechanism. Under incomplete information, pure allocations based only on ordinal rankings of objects with constant transfers are obtained as second best outcomes. Information beyond ranking can only be exploited if wealth levels are known and wealth is adjusted in such a way that ex-post wealth of every agent is made constant. We furthermore show that banning money might even not be sufficient to satisfy

\footnotetext{
${ }^{24}$ See e.g. http://www.bbc.com/news/world-europe-23753872

${ }^{25}$ According to the German social security statutes, the health insurance must not decline membership (SGB V 175: "Ausübung des Wahlrechts: (1) Die Ausübung des Wahlrechts ist gegenüber der gewählten Krankenkasse zu erklären. Diese darf die Mitgliedschaft nicht ablehnen [...]").
} 
discrimination-freeness. To also exclude discrimination in cases where money might be used outside a centralized mechanism even the object assignment has to be heavily restricted. The object an agent is assigned to must not depend on other agents' preferences.

We, therefore, find that a violation of moral concerns existing in certain markets is not equivalent to the presence of money. However, requiring discrimination-freeness restricts to what extent a mechanism can evaluate preferences and with it the use of transfers. The restrictions exceed the exclusion of transfers if there is a use of money outside the mechanisms to improve the access to resources. Thus, instead of exogenously banning transfers, market designers should rather be aware of the implications of moral concerns behind the desire to ban transfers.

The eligible mechanisms we obtain when discrimination-freeness is a constraint appear to be relevant in many markets. Various mechanisms especially in health and education markets are designed in such a way that the good consumed only depends on an ordinal ranking and that the payment is a lump-sum or solely income dependent. Our model explains the very restricted use of monetary transfers based on the concern of discrimination. It illustrates as well why some mechanisms even restrict the object assignment though not using transfers.

Our work also reveals that some currently used mechanisms are apparently not aligned with social preferences for discrimination-freeness. Within school choice applications, for instance, if better schools are rather in more expensive neighborhoods, living in a rather expensive neighborhood already implies better access to schools. There are indeed claims for rethinking the current system. The chairman of the Black Allicance for Educational Option wrote in a New York Times letter: "If access to high-performing schools has to come down to a number, better it be a lottery number than a ZIP code." 26 Transferring our results to cases where the market participation is associated with costs - as it is claimed to be the case for organ donors - our model points out that discrimination occurs if costs are not reimbursed. Otherwise the decision to donate or not might depend on the wealth status. (Gill et al., 2014) show that in the US, where currently donors basically must not receive any non-medical compensation, wealthier people donate at a much higher rate. Ireland, for instance, recently decided to reimburse the cost of a donation.

This paper is a first step to analyze the implications of moral concerns behind a desire to ban monetary transfers. There is a branch of further research questions that remains open. For instance, in the introduction we only touched the question on which markets discrimination-freeness is desired and why. Furthermore, we did not

\footnotetext{
${ }^{26}$ see http://www.nytimes.com/2011/12/12/opinion/does-school-choice-improve-education.html.
} 
yet consider any trade-offs between discrimination-freeness and efficiency. Knowing more about how preferences depend on wealth in real world applications could help to further differentiate our results. Even if discrimination is a major concern, there also might be further moral concerns beyond discrimination-freeness. Slippery-slope effects are often feared in the context of an introduction of monetary transfers even if they are small and regulated. Another concern mentioned is the exploitation of people in a sense that financial distress might make people unable to decide in their best interest and thus they might regret a decision later. Zargooshi (2001) surveyed people in Iran that sold their kidney after some years. A striking $85 \%$ percent of the questioned people indicated that they regret the donation.

\section{A Appendix}

\section{A.1 Proof of Proposition 1}

To prove Proposition 1 we are interested in how much agents are willing to pay for an object improvement after the assignment $\varphi$ is performed. We first prove an auxiliary lemma showing that if we fix a type profile and vary only the wealth endowment of one agent there is a minimum amount and a maximum amount that each agent is willing to pay for any object improvement when facing her assignment of any $\varphi$ that is not wealth-adjusting.

Lemma 5. Let $\varphi=(\sigma, m)$ be a social choice function that is not wealth-adjusting. Fix any agent $i, \theta_{i}$ and $t_{-i}$ and define $A_{j}\left(e_{i}\right)=e_{j}+m_{j}\left(\theta_{i}, e_{i}, t_{-i}\right)$. There exists $0<\underline{M}<\bar{M}$ such that for all agents $j \neq i$ preferring any object $a \in \Omega$ over $b \in \Omega$

$$
\begin{aligned}
& u\left(a, A_{j}\left(e_{i}\right)-\bar{M}, \theta_{j}\right)<u\left(b, A_{j}\left(e_{i}\right), \theta_{j}\right) \text { for all } e_{i} \in \mathbb{R} \\
& u\left(a, A_{j}\left(e_{i}\right)-\underline{M}, \theta_{j}\right)>u\left(b, A_{j}\left(e_{i}\right), \theta_{j}\right) \text { for all } e_{i} \in \mathbb{R}
\end{aligned}
$$

Proof of lemma. We keep $i, \theta_{i}$ and $t_{-i}$ fix (and therefore omit it in the following) and vary only agent $i$ 's wealth endowment $e_{i}$. The set of agents and the set of objects is finite. Therefore it is sufficient to show that for any agent $j$ preferring any object $a$ over object $b$ we can find an $\bar{M}$ and $\underline{M}$ such that (A.1) and (A.2) hold ( $\underline{M}$ then might depend on $j, a$ and $b$ but we finally we can take the maximum and minimum over all agents and objects to define $\bar{M}$ and $\underline{M}$, respectively). Since $\varphi$ is not wealth-adjusting there exists $\underline{A}$ and $\bar{A}$ such that ex-post wealth $A_{j}\left(e_{i}\right)=e_{j}+m_{j}\left(e_{i}\right) \in[\underline{A}, \bar{A}]$ for all $e_{i}$. The lemma is shown to hold if we find $\underline{M}$ and $\bar{M}$ such that $u\left(a, A-\bar{M}, \theta_{j}\right)<u\left(\theta_{j}, b, A\right)$ and $u\left(a, A-\underline{M}, \theta_{j}\right)>u\left(b, A, \theta_{j}\right)$ for all $A \in[\underline{A}, \bar{A}]$. 
Definition of $\bar{M}$ : Consider any $m>0$. Then, there exists a wealth level $A^{*}$ such that $u\left(a, A^{*}, \theta_{j}\right)>u\left(b, A^{*}-m, \theta_{j}\right)$. Without loss of generality we can choose $A^{*}$ small enough such that $A^{*}<\underline{A}$. We now define $\bar{M}=\bar{A}-A^{*}+m$. By definition of $A^{*}$, $\bar{M}>0$ holds. Then for all $A \in[\underline{A}, \bar{A}]$

$$
u\left(b, A, \theta_{j}\right)>u\left(b, A^{*}, \theta_{j}\right)>u\left(a, A^{*}-m, \theta_{j}\right)=u\left(a, \bar{A}-\bar{M}, \theta_{j}\right)>u\left(a, A-\bar{M}, \theta_{j}\right) .
$$

Definition of $\underline{M}$ : The income effects we imposed on $u$ imply that for any $A$ there exists $M>0$ such that $u\left(a, A-M, \theta_{j}\right)<u\left(b, A, \theta_{j}\right)$. As $u$ is continuous and strictly increasing in $A$ and $u\left(a, A, \theta_{j}\right)>u\left(b, A, \theta_{j}\right)$ we can find an unique $\underline{M}(A)>0$ such that $u\left(a, A-\underline{M}(A), \theta_{j}\right)=u\left(b, A, \theta_{j}\right)$. Furthermore, $\underline{M}(A)$ is continuous in $A$. Then

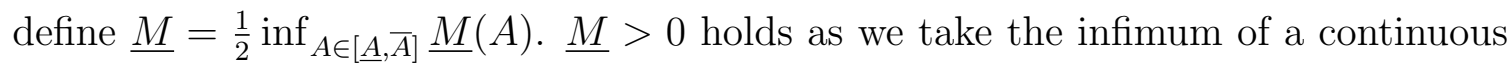
function that is larger than zero over a compact interval. Then for all $A \in[\underline{A}, \bar{A}]$ the inequality $u\left(a, A-\underline{M}, \theta_{j}\right)>u\left(b, A, \theta_{j}\right)$ holds.

This proves the lemma.

$\varphi^{\prime}$ discriminates. We now consider any social choice function $\varphi^{\prime}$ that is not wealthadjusting and assume that $\sigma$ is not Pareto-dominated by any $\sigma^{\prime}$. We show that if $\varphi^{\prime}$ Pareto-dominates $\varphi$ and has the same budget as $\varphi$ it discriminates. To prove this we assume the contrary, i.e. that $\varphi^{\prime}$ is discrimination-free and show that this assumption leads to a contradiction. We select an agent $i$ that received a worse object under $\varphi^{\prime}$ than under $\varphi$ for some type profile $t=\left(t_{i}\right)_{i \in I}$. Such an agent exists because if for all type profiles nobody faced an object impairment under $\varphi^{\prime}$ compared to $\varphi$ and furthermore $\varphi^{\prime}$ Pareto-dominates $\varphi$ and has the same budget, then $\sigma^{\prime}$ would already Pareto-dominate $\sigma$. However, $\sigma$ was selected such that it is not Pareto-dominated by any $\sigma^{\prime}$.

In the following we will fix every attribute of $t$ except for agent $i$ 's wealth level. Therefore, we will omit every type profile information except $e_{i}$. Assume that agent $i$ 's object assignment under $\varphi$ is $a$, under $\varphi^{\prime}$ it is $b$. Due to discrimination-freeness of $\varphi$ and $\varphi^{\prime}$ this holds for any wealth endowment $e_{i}$ of agent $i$. Pareto-dominance of $\varphi^{\prime}$ implies that for every possible wealth endowment $e_{i}$, agent $i$ has to be compensated for receiving object $b$ instead of $a$ by a monetary transfer $M\left(e_{i}\right)$.

We first show, that the amount $M\left(e_{i}\right)$ that compensates agent $i$ for receiving $b$ instead of $a$ becomes arbitrary large if her wealth increases. Agent $i$ 's compensation $M\left(e_{i}\right)$ has to be such that

$$
u\left(a, e_{i}+m_{i}\left(e_{i}\right), \theta_{i}\right) \leq u\left(b, e_{i}+m_{i}^{\prime}\left(e_{i}\right), \theta_{i}\right)=u\left(b, e_{i}+m_{i}\left(e_{i}\right)+M\left(e_{i}\right), \theta_{i}\right)
$$

By the characteristics of the preferences, for any fixed $M$ there is an $\bar{A}$ large enough 
such that $u\left(a, \bar{A}, \theta_{i}\right)>u\left(b, \bar{A}+M, \theta_{i}\right)$. By the assumption that $\varphi$ is not wealth adjusting, particularly wealth of agent $i$ after $\varphi$ is performed is not bounded above and therefore there exists $e_{i}^{\prime}$ such that $u\left(a, e_{i}^{\prime}+m_{i}\left(e_{i}^{\prime}\right), \theta_{i}\right)<u\left(b, e_{i}^{\prime}+m_{i}\left(e_{i}^{\prime}\right)+M, \theta_{i}\right)$. This implies that $M\left(e_{i}\right) \rightarrow \infty$ for $e_{i} \rightarrow \infty$.

At the same time, the amount of money that is available to compensate agent $i$ is bounded above by $(n-1) \bar{M}$ when varying agent $i$ 's wealth level. This follows by the lemma proved above. Therefore, we can find a wealth endowment $e_{i}$ of agent $i$ such that she cannot be compensated any more by the other agents for the object impairment. Then, $\varphi^{\prime}$ cannot be a Pareto-improvement of $\varphi$ which is contradiction.

$\varphi$ is not efficient. We have to find a type profile $t=\left(t_{i}\right)_{i \in I}$ for which $\varphi(t)$ can be Pareto-improved without exceeding the budget of $\varphi(t)$. Fix any object $a \in \Omega$ and consider a type profile $t=\left(t_{i}\right)_{i \in I}$ such that all agent rank object $a$ first. Since $\sigma$ is assumed to be not Pareto-dominated by any other object assignment there is an agent $i$ such that $\varphi$ assigns object $a$ to agent $i$, i.e. $\sigma_{i}(t)=a$. We now vary agent $i$ 's wealth $e_{i}$ and keep $\theta_{i}$ and $t_{-i}$ fixed (and therefore omit $\theta_{i}$ and $t_{-i}$ in the following). Since $\varphi$ is discrimination-free, agent $i$ is assigned to object $a$ for any wealth levels, i.e $\sigma_{i}\left(e_{i}\right)=a$ for all $e_{i}$. By the lemma above we know that independent of agent $i$ 's wealth level each agent other than agent $i$ is willing to pay at least $\underline{M}$ to receive object $a$ instead of their object assignment. On the other hand, if agent $i$ 's ex-post wealth $A_{i}\left(e_{i}\right)$ is small enough then agent $i$ is willing to accept $\underline{M}$ in turn for any other object than $a$. Since $\varphi$ is not wealth-adjusting there es a wealth endowment $e_{i}^{*}$ such that $A_{i}\left(e_{i}^{*}\right)$ is indeed small enough.

Consider now any agent $j$ and call the object that agent $j$ received $b$. Then both agents $i$ and $j$ are better off if they trade objects in turn for $\underline{M}>0$. Performing this trade is a Pareto-improvement of $\varphi(t)$.

\section{A.2 Proof of Proposition 2}

Throughout the proof we fix $t_{-i}$ and therefore we omit it in the following.

$m_{i}$ is independent of $t_{i}$. Assume that $\varphi$ is discrimination-free and implementable and that agent $i$ 's payment is not type-independent. This means that there exist types $t_{i}=\left(\theta_{i}, e_{i}\right)$ and $t_{i}^{\prime}=\left(\theta_{i}^{\prime}, e_{i}^{\prime}\right)$ with $m_{i}\left(t_{i}\right)<m_{i}\left(t_{i}^{\prime}\right)$. We now construct a type $t_{i}^{*}=\left(\theta_{i}, e_{i}^{*}\right) \in T_{i}\left(\theta_{i}\right)$ that has an incentive to misreport. First, choose $e_{i}^{*}$ such that

$$
u\left(\sigma\left(t_{i}^{\prime}\right), e_{i}^{*}+m_{i}\left(t_{i}^{\prime}\right), \theta_{i}\right)>u\left(\sigma\left(t_{i}\right), e_{i}^{*}+m_{i}\left(t_{i}\right), \theta_{i}\right)
$$

This is feasible due to our assumptions on income effects and $m_{i}\left(t_{i}\right)<m_{i}\left(t_{i}^{\prime}\right)$.

Discrimination-freeness of $\varphi$ implies that types $t_{i}$ and $t_{i}^{*}$ receive the same objects 
$\sigma_{i}\left(t_{i}\right)=\sigma_{i}\left(t_{i}^{*}\right)$ because they only differ in wealth. $\varphi$ is implementable, therefore the transfers for $t_{i}$ and $t_{i}^{*}$ also have to be the same, i.e. $m_{i}\left(t_{i}\right)=m_{i}\left(t_{i}^{*}\right)$. Hence $\varphi\left(t_{i}\right)=$ $\varphi\left(t_{i}^{*}\right)$ holds. Due to inequality (A.3), type $t_{i}^{*}$ has an incentive to misreport because reporting $t_{i}^{\prime}$ yields higher utility than reporting $t_{i}^{*}$. This contradicts implementation. Therefore, $m_{i}$ has to be independent of $t_{i}$.

$\sigma_{i}$ only depends on $r_{i}$. Consider two types $t_{i}$ and $t_{i}^{\prime}$ that represent the same object ranking $r_{i}$, i.e. $t_{i}, t_{i}^{\prime} \in T_{i}\left(r_{i}\right)$. From the first part of the proof we know that $m_{i}\left(t_{i}\right)=m_{i}\left(t_{i}^{\prime}\right)$. Implementability of $\varphi$ implies that $\sigma_{i}\left(t_{i}\right)=\sigma_{i}\left(t_{i}^{\prime}\right)$ because otherwise either $t_{i}$ or $t_{i}^{\prime}$ would have an incentive to deviate. Thus, agent $i$ 's object assignment only depends on her rank order list of objects.

Non-bossiness. Assume that $m$ and $\sigma$ are non-bossy. Consider two agents $i$ and $j$. Since $m_{j}$ is independent of $t_{j}$, non-bossiness implies that $m_{i}$ is also independent of $t_{j}$. Repeating the argument for all agents other than $i$ implies that $m_{i}(t)=m_{i}\left(t^{\prime}\right)$. The same then applies for the dependence of $\sigma_{i}$ on $r_{i}$.

\section{A.3 Proof of Proposition 3}

Again we fix $t_{-i}$ throughout the proof and therefore omit it in the following. It is sufficient to show that $m_{i}$ is independent of $\theta_{i}$. Then it follows by the proof of Proposition 2 that $\sigma_{i}$ only depends on rank order lists.

Assume that $\varphi$ is discrimination-free and implementable and that ex-post wealth is not constant (see Proposition). We show that assuming that $m_{i}$ is not independent of $\theta_{i}$ results in a contradiction. For this, we construct a preference profile $\theta_{i}^{*}$ such that there are two types $t_{i}, t_{i}^{\prime} \in T_{i}\left(\theta_{i}^{*}\right)$ that only differ in their wealth level but result in different object allocations. This then contradicts discrimination-freeness and therefore, $m_{i}$ has to be independent of $\theta_{i}$.

Construction of $\theta_{i}^{*}$. If $m_{i}$ is not independent of $\theta_{i}$ there exists $e_{i}, \theta_{i}$ and $\theta_{i}^{\prime}$ such that $m_{i}\left(\theta_{i}, e_{i}\right)<m_{i}\left(\theta_{i}^{\prime}, e_{i}\right)$. Choose $e_{i}^{\prime}$ such that $A_{i}=e_{i}+m_{i}\left(\theta_{i}, e_{i}\right) \neq e_{i}^{\prime}+m_{i}\left(\theta_{i}, e_{i}^{\prime}\right)=A_{i}^{\prime}$. Such an $e_{i}^{\prime}$ exists because ex-post wealth is not constant.

In the following it is convenient to consider choice sets of agents given their wealth endowment. A choice set $C\left(e_{i}\right)$ is the set of all bundles of objects and ex-post wealth available to an agent $i$ with wealth endowment $e_{i}$ by varying her report $\left(t_{-i}\right.$ is still fixed), i.e.

$$
C\left(e_{i}\right)=\left\{\left(\sigma_{i}\left(\theta_{i}, e_{i}\right), m_{i}\left(\theta_{i}, e_{i}\right)+e_{i}\right) \mid \theta_{i} \in \Theta_{i}\right\} .
$$

Implementability of $\varphi$ implies that two different bundles in $C\left(e_{i}\right)$ need to differ in their object (otherwise $\varphi$ cannot be implementable) and therefore $C\left(e_{i}\right)$ contains at most $k$ bundles. Furthermore, define $a=\sigma_{i}\left(\theta_{i}, e_{i}\right)$ and $b=\sigma_{i}\left(\theta_{i}^{\prime}, e_{i}\right) . a \neq b$ holds because 
$\varphi$ is implementable and $m_{i}\left(\theta_{i}, e_{i}\right)<m_{i}\left(\theta_{i}^{\prime}, e_{i}\right)$. Then, for a wealth endowment $e_{i}$ the bundles $\left(a, A_{i}\right)$ and $\left(b, A_{i}+x\right)$ with $x>0$ are in the choice set $C\left(e_{i}\right)$ of agent $i$. On the other hand, for $e_{i}^{\prime}$ the bundles $\left(a, A_{i}^{\prime}\right)$ and $\left(b, A_{i}^{\prime}+x^{\prime}\right)$ with $x^{\prime} \in \mathbb{R}$ are in the choice set $C\left(e_{i}^{\prime}\right)$. This is because if only agent $i$ 's wealth changes, the objects that can be reached by varying the preferences need to be the same due to discrimination-freeness.

Let $\max \left\{C\left(e_{i}\right) \mid \theta_{i}\right\}$ be the most preferred element out of $C\left(e_{i}\right)$ given preferences $\theta_{i}$. If we can construct a preference profile $\theta_{i}^{*}$ such that $\max \left\{C\left(e_{i}\right) \mid \theta_{i}^{*}\right\}$ and $\max \left\{C\left(e_{i}^{\prime}\right) \mid \theta_{i}^{*}\right\}$ entail different objects it implies that $\varphi$ is not discrimination-free. This is because then type $t_{i}=\left(\theta_{i}^{*}, e_{i}\right)$ receives a different object than type $t_{i}^{\prime}=\left(\theta_{i}^{*}, e_{i}^{\prime}\right)$ (due to implementability).

To see how to construct $\theta_{i}^{*}$ first assume that $x^{\prime} \leq 0$. Choose $\theta_{i}^{*}$ such that $a$ is the most, $b$ the second most preferred object and such that $\left(b, A_{i}+x\right)=\max \left\{C\left(e_{i}\right) \mid \theta_{i}^{*}\right\}$ (which is possible as $x \geq 0$ ). However, since $\theta_{i}^{*}$ prefers $a$ over $b$ and $x^{\prime} \leq 0$ we have $u\left(a, A_{i}^{\prime}, \theta_{i}^{*}\right)>u\left(b, A_{i}^{\prime}+x^{\prime}, \theta_{i}^{*}\right)$ which means that somebody with preferences $\theta_{i}^{*}$ chooses a bundle that does not entail object $b$. Now assume that $x^{\prime}>0$. Again choose $\theta_{i}^{*}$ such that $a$ is the most and $b$ the second most preferred object. If $A_{i}<A_{i}^{\prime 27}$ ensure that $\left(a, A_{i}^{\prime}\right)=\max \left\{C\left(e_{i}^{\prime}\right) \mid \theta_{i}^{*}\right\}$ while $u\left(b, A_{i}+x, \theta_{i}^{*}\right)>u\left(a, A_{i}, \theta_{i}^{*}\right)$. As $A_{i}^{\prime} \neq A_{i}$ this is feasible. For $A_{i}>A_{i}^{\prime}$ the general idea is the same and we choose $\theta_{i}^{*}$ such that $\left(a, A_{i}\right)=\max \left\{C\left(e_{i}\right) \mid \theta_{i}^{*}\right\}$ and $u\left(b, A_{i}^{\prime}+x^{\prime}, \theta_{i}^{*}\right)>u\left(a, A_{i}^{\prime}, \theta_{i}^{*}\right)$. This again leads to a different object assignment for types $t_{i}=\left(\theta_{i}^{*}, e_{i}\right)$ and $t_{i}^{\prime}=\left(\theta_{i}^{*}, e_{i}^{\prime}\right)$ which contradicts discrimination-freeness.

\section{A.4 Proof of Proposition 4}

In the following we assume that $\varphi$ is an implementable and discrimination-free social choice function.

Bribe-proofness $\Leftrightarrow$ externality-freeness: It is straight forward that externalityfreeness implies bribe-proofness: If no agent can influence another agent's assignment it never pays off to pay somebody else to state other preferences. Since $\varphi$ is implementable, no agent has an incentive to misreport. This implies that no bribing incentives exist such that an agent $i$ is bribing herself with $\tau=0$. Thus, $\varphi$ is bribe-proof.

We now show that bribe-proofness implies externality-freeness. To ease notation we denote for an agent of type $t_{i}$ the strict preferences over outcomes by $P_{i}$, the weak preferences by $R_{i}$ and indifferences by $I_{i}$.

\footnotetext{
${ }^{27}$ The case differentiation is not necessary for the proof. However, it shows that $\theta_{i}^{*}$ can be constructed by using a utility function $u_{i}^{*}$ of the form $u\left(\omega, A_{i}, \theta_{i}^{*}\right)=v\left(\omega, \theta_{i}^{*}\right)+w\left(A_{i}, \theta_{i}^{*}\right)$ with decreasing marginal value of money.
} 
First, we show that for any agent $i$ another agent $j$ 's report does not influence her utility, i.e. $\varphi_{i}\left(t_{j}, t_{-j}\right) I_{i} \varphi_{i}\left(t_{j}^{\prime}, t_{-j}\right)$. Assume that it does not hold such that there is $t=\left(t_{i}\right)_{i \in I}$ and $t_{j}^{\prime}$ with $\varphi_{i}\left(t_{j}^{\prime}, t_{-j}\right) P_{i} \varphi_{i}\left(t_{j}, t_{-j}\right)$. We show that this assumption produces a contradiction because we can find a type profile such that bribing incentives exist. Continuity of the preferences in money implies the existence of $\delta>0$ such that $\left(\sigma_{j}\left(t_{j}^{\prime}\right), m_{i}\left(t_{j}^{\prime}\right)-\delta\right) P_{i} \varphi_{i}\left(t_{j}, t_{-j}\right)\left(i\right.$ would pay $\delta$ to change type $t_{j}$ 's report from $t_{j}$ to $\left.t_{j}^{\prime}\right)$. Implementability of $\varphi$ implies $\varphi_{j}\left(t_{j}\right) R_{j} \varphi_{j}\left(t_{j}^{\prime}\right)$ and discrimination-freeness of $\varphi$ implies $m_{j}\left(t_{j}\right)=m_{j}\left(t_{j}^{\prime}\right)$. We now change agent $j$ 's wealth level such that she rather prefers taking $\delta$ than reporting truthfully, i.e. we choose $e_{j}^{*}$ such that $\left(\sigma_{j}\left(t_{j}^{\prime}\right), m_{i}\left(t_{j}^{\prime}\right)+\right.$ $\delta) P_{j}^{*} \varphi_{j}\left(t_{j}\right)$. This is possible as $m_{j}\left(t_{j}\right)=m_{j}\left(t_{j}^{\prime}\right)$ and if agent $j$ is poor enough she prefers the difference in money over an object improvement. However, $\varphi\left(t_{j}^{*}\right)=\varphi\left(t_{j}\right)$ (Proposition 2). Now we consider two cases. If $\varphi_{i}\left(t_{j}^{*}, t_{-j}\right) P_{i} \varphi_{i}\left(t_{j}, t_{-j}\right)$ type $t_{i}$ has an incentive to bribe type $t_{j}$ to report $t_{j}^{*}$. Then, any bribing amount is enough for this bribe as $t_{j}$ is indifferent regarding reporting $t_{j}$ or $t_{j}^{*}$. If $\varphi_{i}\left(t_{j}, t_{-j}\right) R_{i} \varphi_{i}\left(t_{j}^{*}, t_{-j}\right)$ agent $i$ has an incentive to bribe agent $j$ of type $t_{j}^{*}$ to report $t_{j}^{\prime}$ with $\tau=\delta$.

Second, we show that $\varphi_{i}\left(t_{j}, t_{-j}\right) I_{i} \varphi_{i}\left(t_{j}^{\prime}, t_{-j}\right)$ implies $\varphi_{i}\left(t_{j}, t_{-j}\right)=\varphi_{i}\left(t_{j}^{\prime}, t_{-j}\right)$. For this assume that $\left(a, m_{1}\right)=\varphi_{i}\left(t_{j}, t_{-j}\right) \neq \varphi_{i}\left(t_{j}^{\prime}, t_{-j}\right)=\left(b, m_{2}\right)$. Then, $a \neq b$ and $m_{1} \neq m_{2}$ (otherwise, agent $i$ cannot be indifferent). Without loss of generality assume that $m_{1}>m_{2}$. Now change agent $i$ 's wealth endowment from $e_{i}$ to $e_{i}^{*}$ such that $\left(a, m_{1}\right) P_{i}^{*}\left(b, m_{2}\right)$. Discrimination-freeness and implementability implies that $\varphi\left(t_{i}^{*}, t_{j}, t_{-i j}\right)=\left(a, m_{1}\right)$. Furthermore, $\varphi\left(t_{i}^{*}, t_{j}, t_{-i j}\right) I_{i}^{*} \varphi\left(t_{i}^{*}, t_{j}^{\prime}, t_{-i j}\right)$ and $\varphi\left(t_{i}^{*}, t_{j}^{\prime}, t_{-i j}\right)=$ $\varphi\left(t_{i}^{*}, t_{j}, t_{-i j}\right)=\left(b, m_{2}\right)$. This is a contradiction as we chose $A_{i}^{*}$ such that $\left(a, m_{1}\right) P_{i}^{*}\left(b, m_{2}\right)$.

Finally, it remains to show that if $\varphi_{i}\left(t_{j}, t_{-j}\right)=\varphi_{i}\left(t_{j}^{\prime}, t_{-j}\right)$ holds for all agents $j$ and all types $t_{i}, t_{-i}, t_{-i}^{\prime}$ it already implies that $\varphi_{i}\left(t_{i}, t_{-j}\right)=\varphi_{i}\left(t_{i}, t_{-j}^{\prime}\right)$. This follows by induction.

Non-bossy social choice functions: By the first part of the proposition externalityfreeness is equivalent to bribe-proofness. Furthermore, bribe-proofnees implies that discrimination-freeness under bribes is preserved. Thus, it remains to show that if $\varphi$ is non-bossy and preserves discrimination-freeness under bribes, then $\varphi$ has to be bribe-proof.

Assume that this is not the case, i.e. that $\varphi$ is not bribe-proof. Then, there exists $t=\left(t_{i}\right)_{i \in N}$ such that $i$ has an incentive to bribe $j \neq i$. Now fix any $m>0$. Change agent $i$ 's endowment from $e_{i}$ to $e_{i}^{\prime}$ such that she is not willing to pay $m$ to change $j$ 's report and $j$ is not willing to accept $m$ to change her report (possible due to non-bossiness). Then for $t_{i}^{\prime}$ and $t_{j}$ the object allocation under any $\varphi_{i j}^{B}$ differs as $i$ won't bribe $j$ any more. This is a contradiction. 


\section{A.5 Proof of Corollary 4}

We show the corollary by showing that an agent facing a choice set of size $j$ randomly chosen out of a set $\Omega$ is facing decreasing expected utility gains. For any agent $i$ let $Z_{j}$ denote the random variable that describes the element with maximal utility of a randomly chosen subset of $\Omega$ of size $j$. Let $\mathbb{E}\left(Z_{j}\right)$ denote the expected utility of $Z_{j}$ for agent $i$. We have to show that the marginal utility gain of raising $j$ is decreasing meaning that

$$
\mathbb{E}\left(Z_{j+1}\right)-\mathbb{E}\left(Z_{j}\right) \leq \mathbb{E}\left(Z_{j}\right)-\mathbb{E}\left(Z_{j-1}\right)
$$

We order the objects with respect to the valuation of the objects, $a_{1}$ denotes the object with the lowest valuation, $a_{k}$ the object with the highest valuation. We consecutively draw objects out of the set $\left\{a_{1}, \ldots, a_{n}\right\}, Y_{j}$ denotes the random variable representing the j-th draw. Then we can write the random variable $Z_{j}$ as $Z_{1}=Y_{1}$ and $Z_{j}=$ $\max \left\{Y_{j}, Z_{j-1}\right\}$ for $j>1$. By using conditional expectation it is then sufficient to show that

$$
\mathbb{E}\left[\left(Z_{j}-Z_{j-1}\right) 1_{Y_{1}=a_{i_{1}}, \ldots, Y_{j-1}=a_{i_{j-1}}}\right] \geq \mathbb{E}\left[\left(Z_{j+1}-Z_{j}\right) 1_{Y_{1}=a_{i_{1}}, \ldots, Y_{j-1}=a_{i_{j-1}}}\right]
$$

for any possible sequence of draws $a_{i_{1}}, \ldots, a_{i_{j-1}}$. However, this just depends on the value of $Z_{j-1}$ and therefore it is sufficient to prove this for $j=2$. This can be done by explicit calculation. 


\section{References}

Abdulkadiroğlu, A. and T. Sönmez (2003). School choice: A mechanism design approach. American Economic Review 93(3), 729-747.

Ambuhl, S., M. Niederle, and A. E. Roth (2015). More money, more problems? Can high pay be coercive and repugnant? Forthcoming in American Economic Review, Papers and Proceedings.

Becker, G. and J. Elias (2007). Introducing incentives in the market for live and cadaveric organ donations. The Journal of Economic Perspectives 21(3), 3-24.

Black, S. (1999). Do better schools matter? Parental valuation of elementary education. The Quarterly Journal of Economics 114(2), 577.

Bogomolnaia, A. and H. Moulin (2001). A new solution to the random assignment problem. Journal of Economic Theory 100(2), 295-328.

Frey, B. S. and F. Oberholzer-Gee (1997). The cost of price incentives: An empirical analysis of motivation crowding-out. The American Economic Review, 746-755.

Gale, D. and L. S. Shapley (1962). College admissions and the stability of marriage. American Mathematical Monthly, 9-15.

Gibbard, A. (1973). Manipulation of voting schemes: a general result. Econometrica, $587-601$.

Gill, J., J. Dong, and J. Gill (2014). Population income and longitudinal trends in living kidney donation in the united states. Journal of the American Society of Nephrology 58(58), 0-75.

Gneezy, U. and A. Rustichini (2000). A fine is a price. Journal of Legal Studies 29, 1.

Hylland, A. and R. Zeckhauser (1979). The efficient allocation of individuals to positions. The Journal of Political Economy, 293-314.

Mellström, C. and M. Johannesson (2008). Crowding out in blood donation: was titmuss right? Journal of the European Economic Association 6(4), 845-863.

Richard, T. (1970). The gift relationship: From human blood to social policy. Allen and Unwin London;. 
Roth, A. E. (2007). Repugnance as a constraint on markets. Journal of Economic Perspectives $21(3), 37-58$.

Sandel, M. (2012). What money can't buy: the moral limits of markets. Farrar, Straus and Giroux.

Satterthwaite, M. and H. Sonnenschein (1981). Strategy-proof allocation mechanisms at differentiable points. The Review of Economic Studies 48(4), 587-597.

Satterthwaite, M. A. (1975). Strategy-proofness and arrow's conditions: Existence and correspondence theorems for voting procedures and social welfare functions. Journal of Economic Theory 10(2), 187-217.

Satz, D. (2010). Why some things should not be for sale: the moral limits of markets. Oxford University Press, USA.

Schummer, J. (2000). Eliciting preferences to assign positions and compensation. Games and Economic Behavior 30(2), 293-318.

Sönmez, T. and M. Unver (2011). Matching, allocation, and exchange of discrete resources. Handbook of Social Economics.

Thomson, W. (2014). Non-bossiness. RCER Working Papers 586, University of Rochester - Center for Economic Research.

Todaro, M. and S. Smith (2003). Economic development, 8th edition. Addison Wesley.

Zargooshi, J. (2001). Quality of life of iranian kidney. The Journal of Urology 166(5), 1790-1799. 